Ad Ed-Lib.
A98

EDUCATION

LIBRARY

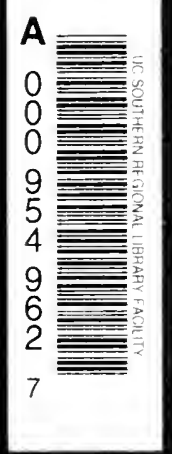




\section{UNIVERSITY OF CALIFORNIA AT LOS ANGELES}
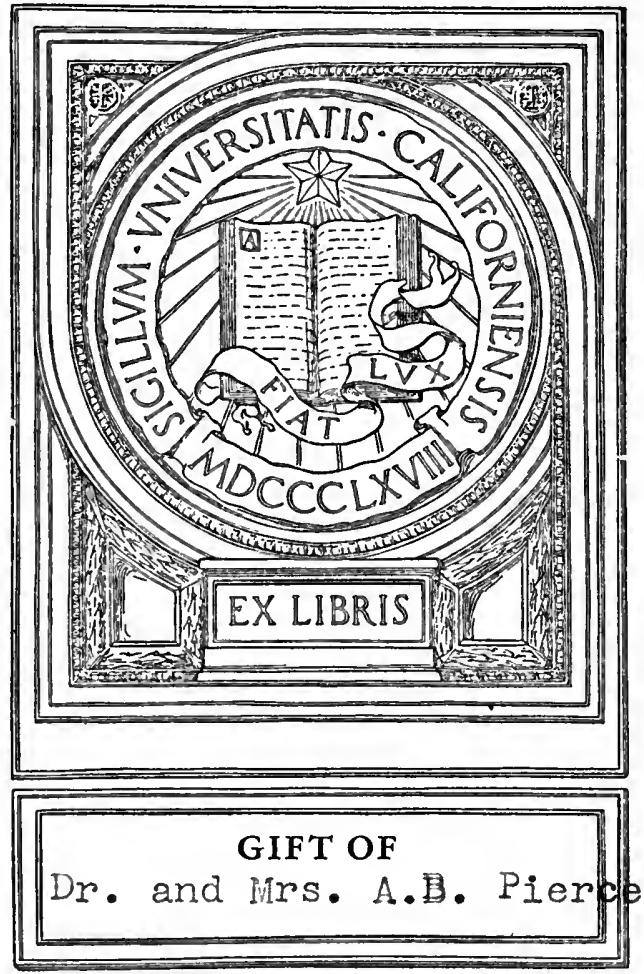
( added La 219

a. 90 
Digitized by the Internet Archive in 2007 with funding from Microsoft Corporation 


\section{AN INDEX NUMBER FOR STATE SCHOOL SYSTEMS}


RANKS OF STATES AS SHOWN BY INDEX NUMBERS FOR FOUR PERIODS

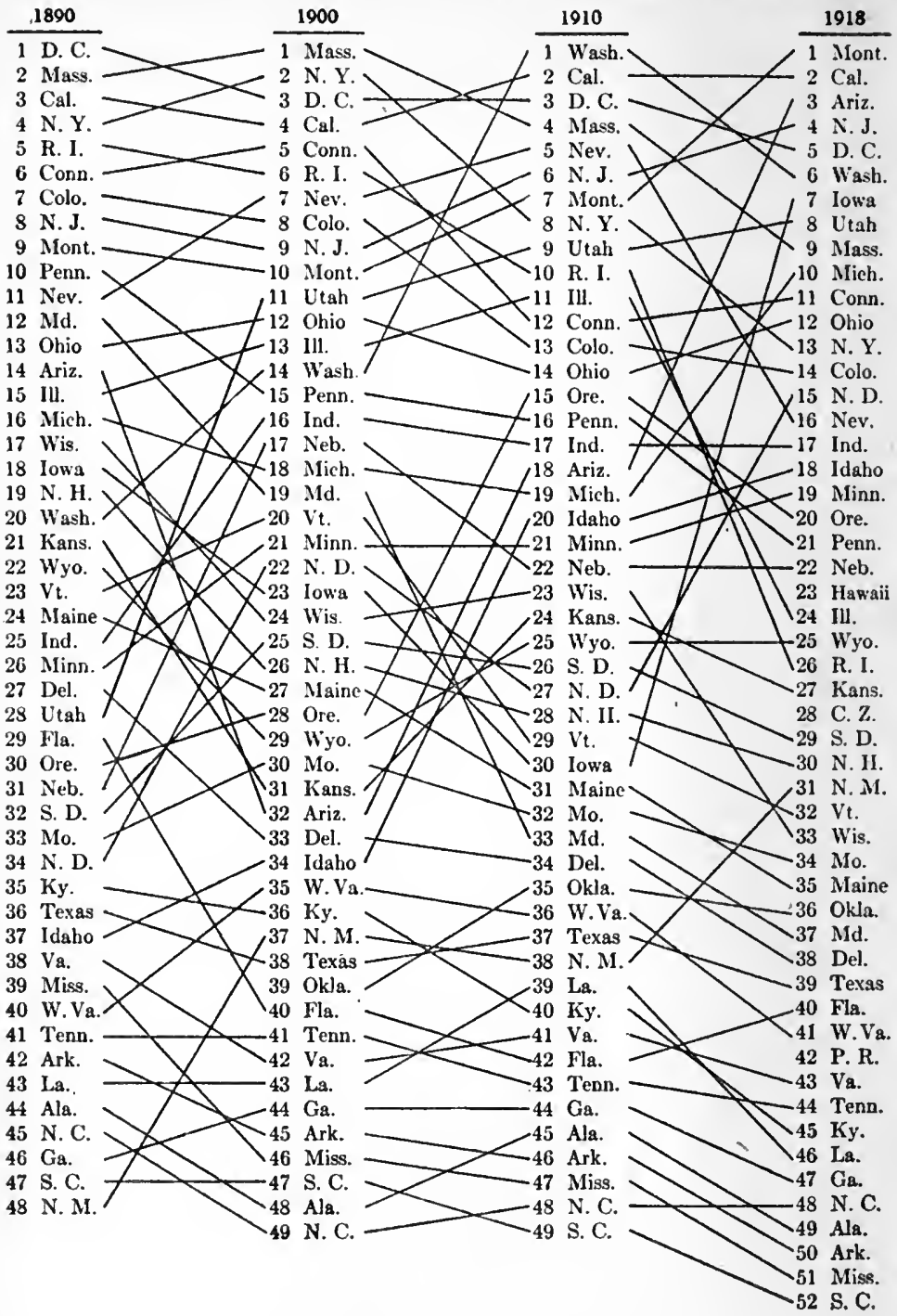




\title{
AN INDEX NUMBER FOR STATE SCHOOL SYSTEMS
}

\author{
LEONARD P. AYRES
}

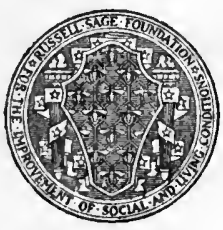

DEPARTMENT OF EDUCATION RUSSELL SAGE FOUNDATION 130 EAST TWENTY-SECOND STREET NEW YORK CITY 
Copyright, 1920, BY

TEE RUSSELL SAGE FOUNDATION

Printed April, 1920: 2,000 copies

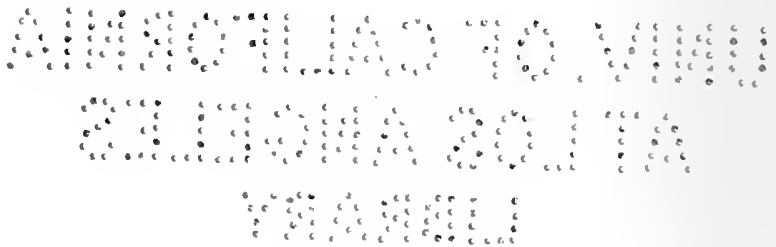

WM - F. PELL CO - PRINTERS

PHILADELPGIA

"ैy 


\section{TABLE OF CONTENTS}

(a) List of Tables

Changes in Ten Years

$\gamma$ Index Numbers

An Index Number for State School Systems 14

What Items of 100 Each Would Imply

The Computation of the Index Number

Data for 48 Years

Groups of States

Standing of the States in 1890

Standing of the States in 1900

Standing of the States in 1910

Standing of the States in 1918

36

Hawaii, the Canal Zone, and Porto Rico

Education in Porto Rico

38

38

Standing of the States in Four Decades 42

Standing of the States During Five Periods 46

The 48 States in 1918

Gains and Losses in 28 Years

Index Numbers Stated as Whole Numbers 52

Educational Results and Financial Expenditures

ע) Significance of the Index Number 57

of Accuracy of Data 
Private and Parochial Schools

Different Types of States

Purchasing Power of Money

62

High School Data

63

Teachers' Salaries

63

Limitations

65

Summary

66

68 


\section{LIST OF TABLES}

TABIE

PAGE

1. Components and index numbers. United States. 1871 to 1918

2. Index numbers for divisions. Seven periods

3. Components and index numbers of states. 1890

4. Components and index numbers of states. 1900

5. Components and index numbers of states. 1910

6. Components and index numbers of states. 1918

7. Index numbers of states at four periods

8. Ranks of states as shown by index numbers for four periods

9. Ranks of states as shown by index numbers for five periods

10. Rank of each state in each eomponent of the index in 1918

11. Changes in index numbers and in ranks of states. 1890 to 1918

12. Index numbers of states in 1918

13. Sum of five educational components and sum of five financial eomponents of index number for each state in 1918 



\section{AN INDEX NUMBER FOR STATE SCHOOL SYSTEMS}

During the past fifty years the United States Bureau of Education has published annual reports setting forth conditions in the public schools of each state in the Union. Each year it has gathered and made public the figures which measure certain of the larger and more important phases of the educational effort and attainment of the states. Included in these records are the figures which show how many of the children are attending the public schools and for how many days during the year, what numbers of the children are in the higher schools, and what price in dollars the state is paying to purchase this education for them. These figures reflect the diffusion, the amount, and the quality of the education that the children are receiving.

This collection of data is of unique value. There are few, if any, other governmental ac- 
tivities for which so many significant facts have been gathered, by uniform methods, over so long a period of time. Certain of the fundamental records of the Bureau that run back in unbroken series for half a century contain a body of trustworthy information of the greatest value and of such a nature that, if the data had not been preserved, they could not now be collected by any agency or by any expenditure of effort and money.

During the winter of 1919-20, the Department of Education of the Russell Sage Foundation has been engaged in the task of selecting, from this mass of statistical material, figures that could be combined into a statistical measurement of the accomplishments of the school systems in the several states.

\section{Changes in Ten Years}

The method which has been followed may be illustrated by considering at this point two sets of figures showing certain phases of educational conditions in the public schools of the nation in the years 1900 and 1910. The data are for the United States as a whole and, for the sake of simplicity, they are here set down as whole numbers with their decimal fractions omitted. They are as follows: 
1. Per cent of school population attending school daily

2. Average days attended by each child of school age

3. Average number of days schools were kept open

4. Per cent that high school attendance was of total attendance

5. Per cent that boys were of girls in high schools

6. Average expenditure per child in average attendance

19001910

$\$ 20 \$ 33$

7. Average expenditure per child of school age

8. Average expenditure per teacher employed

9. Expenditure per pupil for purposes other than teachers' salaries

10. Expenditure per teacher employed for salaries

$\$ 10 \$ 18$

$\$ 508 \$ \$ 15$

$\$ 7 \quad \$ 13$

$\$ 325 \$ 485$

These two sets of figures reflect in convincing manner improvements that took place in the administration of public education during the decade from 1900 to 1910. In every instance the figure for the later date is greater than that for the earlier one and in several cases the increase is most marked.

The proportion of children attending school increased, the school year was lengthened, the proportion of children attending high schools was enlarged, and the funds expended for the support of education were greatly augmented. These are true indicators of improved conditions in the school system and it is by com- 
bining these measurements, by methods that will be explained, that an index number has been constructed for measuring the status of the public school systems of the states.

\section{INDEX Numbers}

The index number is a well established statistical device commonly used for measuring changes in wholesale and retail prices and rates of wages over long periods of time. Such a number may be constructed by securing each month the prices of a uniform list of commodities at a selected and unchanging list of establishments, and then computing the average price for the whole list for each month. As this average rises or falls it reflects the general changes taking place in the prices of the types of commodities represented. If some of the commodities are more important than others, or are commonly consumed in greater quantities, methods of weighting are used to make sure that each element shall exercise only its proper share of influence in the final result.

Such numbers are commonly reduced to percentages and the number for a given month is stated as being so many per cent of the figure for some previous period. Thus whole- 
sale prices are commonly given as percentages of the average price for the decade from 1890 to 1900 , when they reached the lowest point recorded. In a similar way the index number for the cost of living, compiled by the Unitéd States Bureau of Labor Statistics, takes the price in 1913 as 100 and states its figure from month to month on that basis. By the beginning of 1920 this index number had risen to 199 .

There are several well established index numbers for the prices of investment stocks and in these cases there is usually no method of weighting employed, nor are the figures commonly stated in terms of a per cent variation from the figure for a determined point of departure. The reason for this is that the unit price of each stock is commonly 100 and the different stocks are considered as being of approximately equal significance so that weighting is unnecessary.

The 10 sets of educational data that have been considered are unusually well adapted for inclusion in an index number. Increases in them reflect improved educational conditions and decreases reflect worse conditions. The data for these 10 items have been gathered by substantially uniform methods from 
identical establishments (the 48 states) over a long period of years. A third, and most important, characteristic is that each of the items can readily be stated in percentage terms. Like the stock prices they can all be measured in terms of a theoretical par value of 100 , and because of this they can be combined in an index number that is a direct average instead of a relative percentage.

\section{An Index Number for State School SysTeMs}

The 10 sets of data from which the new index number has been constructed are the ones already referred to. These data are the following:

1. Per cent of school population attending school daily.

2. Average days attended by each child of school age.

3. Average number of days schools were kept open.

4. Per cent that high school attendance was of total attendance.

5. Per cent that boys were of girls in high schools.

6. Average annual expenditure per child attending. 
7. Average annual expenditure per child of school age.

8. Average annual expenditure per teacher employed.

9. Expenditure per pupil for purposes other than teachers' salaries.

10. Expenditure per teacher for salaries.

The new index number is the average of the 10 figures corresponding to these 10 headings, after certain of them have been so multiplied or divided by constants as to bring each into comparability with a standard of 100 . For example, the average number of days the schools were kept open in the United States as a whole in 1910 was 157.5. The standard or "par value" length of term is taken as 200 days. Hence the actual number of days for 1910 , or 157.5 , is divided by two, which gives the figure 78.75 as one of the 10 components of the final index number. In a similar way an average monthly salary of $\$ 100$ is taken as the standard for the last of the 10 items. The actual average in 1910 was $\$ 485.22$ for the year, or $\$ 40.44$ for each month and this number is included as one of the components without further change.

The other items are treated by similar methods, and, finally all 10 are added and the 
average taken as the index number. This may be computed for the whole number of states, or any group of them, or any one among them, for any year. When so computed it expresses in a single number the degree to which the average of the 10 different measures taken together approaches the standard of 100 .

The 10 numbered paragraphs which follow tell how the 10 items have been entered in the final index number and show how each one has been brought into relationship with a common basis, or educational par value, of 100. All the figures relate to public day schools.

1. The per cent of school population attending school daily. This item has been included as a percentage figure. It can never exceed 100. It is the per cent that the average daily attendance in day public schools is of the whole number of children of school age. The children of school age are those more than five and less than eighteen years old and these numbers are supplied by the United States Census.

2. Average days attended by each child of school age. This item has been included in the final total as one half of the number found by dividing the aggregate days of attendance by 
the number of children of school age. If all the children of school age attended school every day, and if the schools were all open for 200 days during the year, the days per child would be 200 . In order to make it comparable with the percentage figures, the number, as directly computed, is divided by two.

3. Average number of days schools were kept open. This figure is included in the index as one-half of the figure given in the report of the Bureau of Education. At present a school year of 200 days is exceeded in few cities and by no states. A record of 200 days, if found, would be included in the index as 100. If the school year were extended beyond 200 days, the number added would be proportionally greater than 100 .

4. Per cent that high school attendance was of total attendance. This figure is included as three times the percentage that the high school pupils are of all the pupils attending. Since there are eight elementary grades and four high school grades, only one-third of the pupils could ever be in the high schools even if no children died and none dropped out. Since a perfect record would be made by having $331 / 3$ per cent of the pupils in the high school, the actual percentage found is multi- 
plied by three before being included in the final results. Under present conditions the number so used must always be less than 100 .

5. Per cent that boys were of girls in high schools. This item is included in the final total at its face value. In general many more girls attend high school than do boys, but the proportion of boys is increasing. Its limit is taken as 100 per cent. In the very few instances in which more boys have been recorded than girls, the per cent of girls to boys has been used.

6. Average annual expenditure per child attending. This is entered in dollars at its face value. It is found by dividing the total expenditures by the average daily attendance in public day schools.

7. Average annual expenditure per child of school age. This item is entered in dollars at its face value. It is the total expenditures divided by the number of children of from five to eighteen years of age.

8. Average annual expenditure per teacher employed. This is entered as the result found by dividing the total expenditure by the whole number of teachers and dividing this quotient by 24 . It is thus one-half of the monthly expenditure per teacher if all twelve 
months of the year be taken into consideration.

9. Expenditure per pupil for purposes other than teachers' salaries. This item is entered in the final total as the result found by dividing the expenditures for purposes other than salaries by the number of children in average daily attendance and multiplying this figure by two.

10. Expenditure per teacher for salaries. This is entered as the total expenditure for salaries divided by the whole number of teachers employed during the year and divided by 12 . It gives the average monthly salary per teacher if all twelve months are taken into consideration, and every teacher is included.

\section{What Items of 100 Each Would Imply}

The methods by which the different items are treated before being included in the final index number are not methods of weighting as that term is usually employed. They do not depend on arbitrary judgments or computed ratios as to the comparative degrees of importance that ought to be attached to the several items. The 10 elements entering into the final index number are inter-related and, 
in some measure, inter-dependent. They have been treated by methods which bring each item into comparability with a common theoretical standard of 100 . This may be made clear by showing the conditions that would exist in a state school system having a score of 100 in each of the 10 items. These conditions would be as follows:

Value entered

1. One hundred per cent of the children of school age would attend school and all would have perfect attendance

2. Each child would attend school 200 days each year

3. The school term would be 200 days

4. Thirty-three and one-third per cent of the pupils would be in high school

in index

5. The boys in high school would be equal to 100 per cent of the girls

6. The annual expenditure would average $\$ 100$ for each child in average attendance

7. The annual expenditure would average $\$ 100$ for each child of school age

8. The expenditures would amount to $\$ 200$ per month for each teacher employed

9. The expenditures for purposes other than teaching would amount to $\$ 50$ per year per child attending

10. Teachers' salaries would average $\$ 100$ per month for 12 months in the year

Total divided by 10 - the index number

It will be noted that, while all the items are brought into comparable relation to 100 , this is not a limit for any of them except the first, fourth, and fifth. The other seven items can 
exceed 100 in theory and some of these do exceed that amount in cases that will be recorded further on in this book. They may exceed 100 in the same way that the price of a stock may exceed its par value of 100 .

\section{The Computation of the Index Number}

We may now return to the earlier illustration of the data for the entire United States for 1900 and for 1910. When these figures are converted into terms of the index number they give the results which follow:

1. Per cent of school population attending school daily

2. Average days attended by each child of school age

3. Average number of days schools were kept open

4. Per cent that high school attendance was of total attendance

5. Per cent that boys were of girls in high schools

$\begin{array}{cc}\text { Index } & \text { Index } \\ \text { item } & \text { item } \\ 1900 & 1910\end{array}$

49.68

52.65

35.90

41.46

72.15

78.75

14.65

21.40

71.35

77.85

6. Average expenditure per child attending

7. Average expenditure per child of school age

8. Average expenditure per teacher employed

9. Expenditure per pupil for purposes other than teachers' salaries

10. Expenditure per teacher for salaries

20.21

33.23

$10.04 \quad 17.50$

21.17

33.95

14.54

26.87

27.12

40.44

Average of 10 items - index number

33.68

42.41 
These two sets of figures, reflecting certain phases of educational conditions in the United States as a whole in 1900 and 1910, illustrate the purpose and method of the new index number. Ten phases of educational conditions are measured. The numbers measuring them are brought into comparability with 100 , which is a definable standard of attainment. These 10 numbers are then added and averaged and thus condensed into a single index number. This single number in the present case is 33.68 for the year 1900 and 42.41 for the year 1910. The increase during the period is the difference between them, or 8.73.

This increase represents improvement. It is the average increase in 10 different measurements of school accomplishment, when all 10 measures are expressed in fairly comparable terms. It is a single number expressing the average increase in 10 different measures of the diffusion, the quantity, and the quality of the public education received by the children.

It is not claimed that educational efficiency varies directly with any or all of these measures, or is completely reflected by them. For the present such exact appraisal of effort and accomplishment in education is not attain- 
able. We can, however, secure from the data presented in this volume convincing evidence that there is a real relationship between expenditure and results in education. We cannot prove that the higher paid teacher is individually more skilled than the lower paid one, but it can be shown that as salaries increase, attendance improves, and more pupils are found in the high schools. It can further be shown that as salaries increase, expenditures for purposes other than salaries also increase and this results in better buildings and equipment. It is further evident that, in general, the purely educational items of the index tend to increase as the financial items grow larger even where there is no functional connection between the two sets of data.

\section{DATA FOR 48 YEARS}

In Table 1 , on page 25, data are presented showing the 10 items and the resulting index number for the public schools of the entire United States from 1871 through 1918. In each case the year named is that in which the school year ended. Thus 1918 refers to the school year 1917-18. The 10 items are the ones that have been explained in the preceding pages and they bear the same numbers 
from one to 10 . The final column gives the index number which is the average of the $\mathbf{1 0}$ items. All the data are from the reports of the Federal Bureau of Education. Those for 1918 are from data as yet unpublished but furnished through the courtesy of the Commissioner of Education. The data for 1917 are not as yet available.

Every endeavor has been made to insure the accuracy of the figures here presented. They have been most carefully checked and finally subjected to an independent, duplicate re-computation in the attempt to find and exclude any errors. It will be found, however, that there are certain minor discrepancies between the figures here presented and the official figures in the reports of the Commissioner of Education. Some of these are due to the fact that the Federal Bureau frequently receives new data after the annual report has gone to press and uses them to correct the figures involved when the next report is printed. Other differences are introduced as each new set of Census reports makes it possible to correct the computations of school population. The attempt has been made to take these corrections into account. Because of this the figures are, in some in- 
TABLE 1.-COMPONENTS AND INDEX NUMBERS. UNITED STATES. 1871 TO 1918

\begin{tabular}{|c|c|c|c|c|c|c|c|c|c|c|c|}
\hline Year & 1 & 2 & 3 & 4 & 5 & 6 & 7 & 8 & 9 & 10 & Index \\
\hline $\begin{array}{l}1871 \\
1872 \\
1873 \\
1874 \\
1875 \\
1876 \\
1877 \\
1878 \\
1879\end{array}$ & $\begin{array}{l}36.94 \\
37.08 \\
36.98 \\
38.52 \\
39.14 \\
38.59 \\
38.69 \\
40.28 \\
39.97\end{array}$ & $\begin{array}{l}24.35 \\
24.73 \\
23.87 \\
24.81 \\
25.52 \\
25.68 \\
25.55 \\
26.58 \\
26.02\end{array}$ & $\begin{array}{l}66.05 \\
66.70 \\
64.55 \\
64.40 \\
65.20 \\
66.55 \\
66.05 \\
66.00 \\
65.10\end{array}$ & $\begin{array}{l}1.25 \\
1.48 \\
1.71 \\
1.78 \\
1.83 \\
1.93 \\
1.99 \\
1.97 \\
2.14\end{array}$ & $\begin{array}{l}65.80 \\
65.90 \\
65.90 \\
65.95 \\
66.00 \\
66.00 \\
66.00 \\
66.10 \\
66.20\end{array}$ & \begin{tabular}{|l|}
15.20 \\
15.93 \\
16.06 \\
15.84 \\
15.91 \\
15.70 \\
14.64 \\
13.68 \\
12.97
\end{tabular} & $\begin{array}{l}5.62 \\
5.91 \\
5.94 \\
6.10 \\
6.23 \\
6.06 \\
5.66 \\
5.51 \\
5.18\end{array}$ & \begin{tabular}{|l|}
13.08 \\
13.46 \\
13.38 \\
13.43 \\
13.49 \\
13.34 \\
12.40 \\
11.89 \\
11.33
\end{tabular} & $\begin{array}{r}11.67 \\
12.15 \\
11.93 \\
11.59 \\
10.97 \\
10.48 \\
9.02 \\
7.93 \\
7.34\end{array}$ & \begin{tabular}{|l|}
16.11 \\
16.67 \\
16.75 \\
17.08 \\
17.67 \\
17.75 \\
17.17 \\
16.92 \\
16.25
\end{tabular} & $\begin{array}{l}25.61 \\
26.00 \\
25.71 \\
25.95 \\
26.20 \\
26.21 \\
25.72 \\
25.69 \\
25.25\end{array}$ \\
\hline $\begin{array}{l}1880 \\
1881 \\
1882 \\
1883 \\
1884 \\
1885 \\
1886 \\
1887 \\
1888 \\
1889\end{array}$ & $\begin{array}{l}40.78 \\
39.96 \\
40.32 \\
41.47 \\
43.02 \\
43.51 \\
43.96 \\
43.95 \\
44.35 \\
44.06\end{array}$ & $\begin{array}{l}26.55 \\
25.99 \\
26.45 \\
26.91 \\
27.77 \\
28.43 \\
28.66 \\
28.85 \\
29.34 \\
29.45\end{array}$ & $\begin{array}{l}65.15 \\
65.05 \\
65.60 \\
64.90 \\
64.55 \\
65.35 \\
65.20 \\
65.65 \\
66.15 \\
66.85\end{array}$ & $\begin{array}{l}2.25 \\
2.44 \\
2.65 \\
2.71 \\
3.19 \\
3.70 \\
4.19 \\
4.69 \\
6.60 \\
7.08\end{array}$ & $\begin{array}{l}66.30 \\
66.40 \\
66.50 \\
66.60 \\
66.70 \\
66.80 \\
66.90 \\
67.00 \\
67.10 \\
67.30\end{array}$ & $\begin{array}{l}12.71 \\
13.61 \\
14.05 \\
14.54 \\
14.62 \\
15.11 \\
15.06 \\
15.07 \\
15.72 \\
16.55\end{array}$ & $\begin{array}{l}5.18 \\
5.44 \\
5.66 \\
6.01 \\
6.29 \\
6.57 \\
6.62 \\
6.62 \\
6.97 \\
7.29\end{array}$ & $\begin{array}{l}11.36 \\
11.86 \\
12.45 \\
13.24 \\
13.70 \\
14.11 \\
14.25 \\
14.21 \\
14.91 \\
15.49\end{array}$ & \begin{tabular}{|r|}
7.21 \\
8.34 \\
8.97 \\
9.61 \\
9.87 \\
10.26 \\
9.85 \\
9.67 \\
10.43 \\
11.23
\end{tabular} & $\begin{array}{l}16.27 \\
16.42 \\
16.92 \\
17.75 \\
18.17 \\
18.67 \\
19.17 \\
19.33 \\
19.92 \\
20.42\end{array}$ & $\begin{array}{l}25.38 \\
25.55 \\
25.96 \\
26.37 \\
26.79 \\
27.25 \\
27.39 \\
27.50 \\
28.15 \\
28.57\end{array}$ \\
\hline $\begin{array}{l}1890 \\
1891 \\
1892 \\
1893 \\
1894 \\
1895 \\
1896 \\
1897 \\
1898 \\
1899\end{array}$ & $\begin{array}{l}43.97 \\
44.49 \\
44.61 \\
45.04 \\
45.99 \\
46.72 \\
46.89 \\
47.62 \\
48.01 \\
47.12\end{array}$ & $\begin{array}{l}29.60 \\
30.19 \\
30.54 \\
30.79 \\
32.08 \\
32.59 \\
32.94 \\
33.81 \\
34.33 \\
33.69\end{array}$ & $\begin{array}{l}67.35 \\
67.85 \\
68.45 \\
68.15 \\
69.75 \\
69.75 \\
70.25 \\
71.00 \\
71.50 \\
71.50\end{array}$ & \begin{tabular}{|r|}
7.47 \\
7.56 \\
8.41 \\
8.63 \\
9.36 \\
11.00 \\
11.65 \\
12.21 \\
13.03 \\
13.82
\end{tabular} & $\begin{array}{l}73.44 \\
67.41 \\
68.31 \\
66.93 \\
67.92 \\
69.91 \\
70.98 \\
73.49 \\
72.67 \\
70.61\end{array}$ & $\begin{array}{l}17.23 \\
17.54 \\
18.20 \\
18.59 \\
18.61 \\
18.41 \\
18.75 \\
18.68 \\
18.75 \\
19.38\end{array}$ & $\begin{array}{l}7.58 \\
7.80 \\
8.12 \\
8.37 \\
8.56 \\
8.60 \\
8.79 \\
8.90 \\
9.00 \\
9.13\end{array}$ & $\begin{array}{l}16.09 \\
16.69 \\
17.35 \\
17.87 \\
18.48 \\
18.41 \\
18.89 \\
19.31 \\
19.71 \\
20.23\end{array}$ & $\begin{array}{l}11.94 \\
12.18 \\
12.97 \\
13.49 \\
13.67 \\
12.97 \\
13.57 \\
13.60 \\
13.54 \\
13.71\end{array}$ & $\begin{array}{l}21.03 \\
21.83 \\
22.33 \\
22.75 \\
23.42 \\
23.83 \\
24.42 \\
24.58 \\
25.17 \\
26.00\end{array}$ & $\begin{array}{l}29.57 \\
29.35 \\
29.93 \\
30.06 \\
30.78 \\
31.22 \\
31.71 \\
32.32 \\
32.57 \\
32.50\end{array}$ \\
\hline $\begin{array}{l}1900 \\
1901 \\
1902 \\
1903 \\
1904 \\
1905 \\
1906 \\
1907 \\
1908 \\
1909\end{array}$ & $\begin{array}{l}49.68 \\
48.75 \\
49.66 \\
48.79 \\
49.15 \\
49.05 \\
49.23 \\
49.16 \\
49.39 \\
52.33\end{array}$ & & \begin{tabular}{|}
72.15 \\
71.85 \\
72.35 \\
73.60 \\
73.35 \\
75.45 \\
75.30 \\
75.90 \\
77.05 \\
77.65
\end{tabular} & $\begin{array}{l}14.65 \\
15.18 \\
14.95 \\
16.07 \\
16.85 \\
17.77 \\
18.47 \\
18.88 \\
19.01 \\
19.90\end{array}$ & & $\begin{array}{l}20.21 \\
21.24 \\
21.54 \\
22.88 \\
24.13 \\
25.40 \\
26.29 \\
28.24 \\
30.56 \\
31.66\end{array}$ & $\begin{array}{l}10.04 \\
10.35 \\
10.70 \\
11.16 \\
11.86 \\
12.28 \\
12.94 \\
13.88 \\
15.09 \\
16.57\end{array}$ & $\begin{array}{l}21.17 \\
21.95 \\
22.47 \\
23.42 \\
25.01 \\
26.40 \\
27.57 \\
29.17 \\
31.23 \\
33.03\end{array}$ & $\begin{array}{l}14.54 \\
15.71 \\
15.69 \\
17.22 \\
18.62 \\
19.88 \\
20.71 \\
22.62 \\
24.94 \\
25.92\end{array}$ & $\begin{array}{l}27.12 \\
27.67 \\
28.58 \\
29.17 \\
30.75 \\
32.17 \\
33.33 \\
35.00 \\
37.00 \\
39.08\end{array}$ & $\begin{array}{l}\mathbf{3 3 . 6 8} \\
\mathbf{3 3 . 8 5} \\
\mathbf{3 4 . 2 0} \\
\mathbf{3 4 . 9 1} \\
\mathbf{3 5 . 7 7} \\
\mathbf{3 6 . 9 1} \\
\mathbf{3 7 . 4 1} \\
\mathbf{3 8 . 3 2} \\
\mathbf{3 9 . 6 3} \\
\mathbf{4 1 . 3 7}\end{array}$ \\
\hline $\begin{array}{l}1910 \\
1911 \\
1912 \\
1913 \\
1914 \\
1915 \\
1916 \\
1918\end{array}$ & $\begin{array}{l}52.65 \\
52.02 \\
52.85 \\
53.20 \\
54.68 \\
56.63 \\
57.20 \\
56.20\end{array}$ & $\begin{array}{l}41.46 \\
40.78 \\
41.75 \\
42.05 \\
43.39 \\
45.13 \\
45.85 \\
45.20\end{array}$ & $\begin{array}{l}78.75 \\
78.40 \\
79.00 \\
79.05 \\
79.35 \\
79.70 \\
80.15 \\
80.35\end{array}$ & $\begin{array}{l}21.40 \\
22.96 \\
24.93 \\
25.01 \\
25.72 \\
26.65 \\
28.43 \\
31.78\end{array}$ & $\begin{array}{l}77.85 \\
78.46 \\
79.36 \\
79.42 \\
79.91 \\
82.70 \\
83.06 \\
75.70\end{array}$ & $\begin{array}{l}33.23 \\
34.71 \\
36.26 \\
38.31 \\
39.03 \\
40.47 \\
41.72 \\
49.11\end{array}$ & $\begin{array}{l}17.50 \\
18.06 \\
19.16 \\
20.38 \\
21.34 \\
22.92 \\
23.87 \\
27.58\end{array}$ & $\begin{array}{l}33.95 \\
34.89 \\
36.76 \\
38.43 \\
39.87 \\
41.77 \\
42.89 \\
47.61\end{array}$ & $\begin{array}{l}26.87 \\
27.97 \\
29.76 \\
31.90 \\
32.56 \\
34.85 \\
35.93 \\
44.07\end{array}$ & $\begin{array}{l}40.44 \\
41.58 \\
43.25 \\
44.08 \\
46.50 \\
47.58 \\
48.84 \\
52.50\end{array}$ & $\begin{array}{l}42.41 \\
42.98 \\
44.31 \\
45.18 \\
46.24 \\
47.84 \\
48.79 \\
51.01\end{array}$ \\
\hline
\end{tabular}


stances, slightly different from the figures published by the Federal Bureau for the same year. In most cases these differences are in the decimal places only.

The figures of the last column of Table 1 show that the index number for the United States has made almost unbroken progress during the past half century except during the first decade, from 1871 to 1880 . This decade was a period of severe financial disturbance and depression following the Civil War.

During this period teachers' salaries (column 10) first advanced and then were reduced, expenditures for purposes other than teaching (column 9) were sharply cut down, the school term (column 3) was shortened, and the proportion of children of school age attending (column 1) increased but little. The index number during the period shows a slight falling off.

From this point on the record is one of almost unbroken progress. In general terms it may be stated that the index number doubled within the period, advancing from 26 at the beginning to 51 at the end. 


\section{Groups of States}

The reports of the Bureau of Education have presented their data, not only for the individual states, but also for five great groups of states or divisions. The states constituting these five divisions are the following:

North Atlantic Division

Maine

New Hampshire

Vermont

Massachusetts

Rhode Island

Connecticut

New York

New Jersey

Pennsylvania

North Central Division

Ohio

Indiana

Illinois

Michigan

Wisconsin

Minnesota

Iowa

Missouri

North Dakota

South Dakota

Nebraska

Kansas

South Atlantic Division

Delaware

Maryland

District of Columbia

Virginia

West Virginia

North Carolina

South Carolina

Georgia

Florida 
South Central Division

Kentucky

Tennessee

Alabama

Mississippi

Louisiana

Texas

Arkansas

Oklahoma

Western Division

Montana

Wyoming

Colorado

New Mexico

Arizona

Utah

Nevada

Idaho

Washington

Oregon

California

The index numbers have been worked out for these five divisions for each decade year and 1918. The results are presented in Table 2.

TABLE 2.-INDEX NUMBERS FOR DIVISIONS. SEVEN PERIODS

\begin{tabular}{l|c|c|c|c|c|c}
\hline Year & $\begin{array}{c}\text { North } \\
\text { Atlantic } \\
\text { Divi- } \\
\text { sion }\end{array}$ & $\begin{array}{c}\text { North } \\
\text { Central } \\
\text { Division }\end{array}$ & $\begin{array}{c}\text { South } \\
\text { Atlantic } \\
\text { Division }\end{array}$ & $\begin{array}{c}\text { South } \\
\text { Central } \\
\text { Division }\end{array}$ & $\begin{array}{c}\text { Western } \\
\text { Division }\end{array}$ & $\begin{array}{c}\text { Conti- } \\
\text { nental } \\
\text { United } \\
\text { States }\end{array}$ \\
\hline 1871 & 30.76 & 25.82 & 18.41 & 18.20 & $\mathbf{2 8 . 8 1}$ & $\mathbf{2 5 . 6 1}$ \\
1880 & 30.47 & 27.28 & 18.64 & 17.56 & $\mathbf{3 0 . 6 1}$ & $\mathbf{2 5 . 3 8}$ \\
1890 & 36.54 & 31.20 & $\mathbf{2 1 . 6 7}$ & 21.38 & 35.86 & $\mathbf{2 9 . 5 7}$ \\
1900 & 42.85 & 35.72 & $\mathbf{2 3 . 5 1}$ & 23.05 & 38.16 & $\mathbf{3 3 . 6 8}$ \\
1910 & 50.06 & $\mathbf{4 5 . 5 0}$ & $\mathbf{3 1 . 0 9}$ & 30.31 & 54.68 & $\mathbf{4 2 . 4 1}$ \\
1916 & $\mathbf{5 7 . 0 9}$ & $\mathbf{5 2 . 9 8}$ & $\mathbf{3 4 . 5 4}$ & 34.75 & $\mathbf{6 2 . 8 7}$ & $\mathbf{4 8 . 7 9}$ \\
1918 & 58.94 & 56.66 & $\mathbf{3 4 . 7 6}$ & 35.83 & 64.24 & $\mathbf{5 1 . 0 1}$ \\
\hline
\end{tabular}


The figures of the table indicate that the school systems of the western states have made the greatest progress during the period covered. In all the divisions the most rapid forward movement was made during the decade from 1900 to 1910 . In the eight years from 1910 to 1918 the most noteworthy advance was made by the states of the North Central Division. These states of the Middle West show particularly rapid progress during the last two years of the period. 


\section{Standing of the States In 1890}

In addition to the index numbers for the entire country and for its divisions, the data have been computed for each state for three decade years and the final year. Table 3 gives the figures for the 10 items in the index number for each state and the District of Columbia for the school year ended in 1890 . For convenience of reference the states are arranged in alphabetical order.

In Table 3 the figures are given for only 47 states because the data for Oklahoma did not run back to that year. In this and the similar tables which follow, figures for the District of Columbia have been included, but it should be remembered that they are only partly comparable with the other figures because the District of Columbia mostly consists of one considerable city.

The figures indicate that Massachusetts with an index number of 45.86 was the leading state in 1890, and was followed by California, New York, Rhode Island, and Connecticut in that order. The lowest index numberthat of New Mexico-was 10.02, or distinctly less than one-quarter as large as the number for Massachusetts. 
TABLE 3.-COMPONENTS AND INDEX NUMBERS OF STATES. 1890

\begin{tabular}{|c|c|c|c|c|c|c|c|c|c|c|c|}
\hline State & 1 & 2 & 3 & 4 & 5 & 6 & 7 & 8 & 9 & 10 & Index \\
\hline $\begin{array}{l}\text { Ala. } \\
\text { Ariz. } \\
\text { Ark. } \\
\text { Cal. } \\
\text { Colo. } \\
\text { Conn. } \\
\text { D. C. } \\
\text { Del. } \\
\text { Fla. } \\
\text { Ga. }\end{array}$ & $\begin{array}{l}33.78 \\
31.02 \\
36.95 \\
51.15 \\
42.69 \\
57.06 \\
48.20 \\
41.37 \\
49.82 \\
35.32\end{array}$ & $\begin{array}{l}12.41 \\
19.54 \\
13.86 \\
40.31 \\
30.82 \\
52.07 \\
46.51 \\
32.68 \\
29.89 \\
12.51\end{array}$ & $\begin{array}{l}36.75 \\
63.00 \\
37.50 \\
78.80 \\
72.20 \\
91.25 \\
96.50 \\
79.00 \\
60.00 \\
35.20\end{array}$ & $\begin{array}{r}.62 \\
2.70 \\
2.02 \\
8.00 \\
6.73 \\
14.11 \\
18.59 \\
8.32 \\
2.36 \\
2.18\end{array}$ & $\begin{array}{l}74.42 \\
61.54 \\
76.07 \\
89.39 \\
61.04 \\
65.27 \\
92.93 \\
71.92 \\
97.29 \\
50.72\end{array}$ & $\begin{array}{r}4.88 \\
38.68 \\
6.84 \\
35.38 \\
43.43 \\
25.79 \\
32.14 \\
11.60 \\
7.97 \\
4.17\end{array}$ & $\begin{array}{r}1.65 \\
12.00 \\
2.53 \\
18.10 \\
18.54 \\
14.71 \\
15.49 \\
5.19 \\
3.97 \\
1.47\end{array}$ & $\begin{array}{r}5.87 \\
31.60 \\
8.45 \\
39.77 \\
29.50 \\
21.96 \\
50.66 \\
14.67 \\
8.58 \\
5.33\end{array}$ & $\begin{array}{r}2.52 \\
23.10 \\
1.98 \\
20.80 \\
44.56 \\
18.98 \\
27.88 \\
4.12 \\
12.90 \\
.72\end{array}$ & $\begin{array}{r}8.71 \\
44.30 \\
14.45 \\
56.17 \\
28.72 \\
27.75 \\
57.39 \\
24.12 \\
12.35 \\
9.74\end{array}$ & $\begin{array}{l}18.16 \\
32.75 \\
20.07 \\
43.79 \\
37.83 \\
38.90 \\
48.63 \\
29.30 \\
28.52 \\
15.73\end{array}$ \\
\hline $\begin{array}{l}\text { Idaho } \\
\text { Ill. } \\
\text { Ind. } \\
\text { Iowa } \\
\text { Kans. } \\
\text { Ky. } \\
\text { La. } \\
\text { Maine } \\
\text { Mass. } \\
\text { Md. }\end{array}$ & $\begin{array}{l}41.59 \\
49.80 \\
52.85 \\
53.10 \\
53.97 \\
37.98 \\
24.18 \\
60.49 \\
67.17 \\
33.54\end{array}$ & $\begin{array}{l}14.51 \\
35.36 \\
35.94 \\
39.29 \\
32.38 \\
17.85 \\
12.67 \\
33.87 \\
59.45 \\
31.36\end{array}$ & $\begin{array}{l}34.90 \\
71.00 \\
68.00 \\
74.00 \\
60.00 \\
47.00 \\
52.40 \\
56.00 \\
88.50 \\
93.50\end{array}$ & $\begin{array}{r}2.65 \\
9.23 \\
7.36 \\
11.47 \\
7.13 \\
1.96 \\
2.73 \\
17.06 \\
20.95 \\
3.23\end{array}$ & $\begin{array}{l}64.71 \\
57.49 \\
61.71 \\
62.75 \\
67.87 \\
82.93 \\
45.82 \\
82.84 \\
73.84 \\
84.93\end{array}$ & $\begin{array}{r}17.79 \\
21.63 \\
15.33 \\
20.84 \\
20.44 \\
9.78 \\
8.60 \\
13.50 \\
30.26 \\
18.67\end{array}$ & $\begin{array}{r}7.40 \\
10.77 \\
8.10 \\
11.07 \\
11.03 \\
3.71 \\
2.15 \\
8.16 \\
20.32 \\
6.26\end{array}$ & $\begin{array}{r}14.17 \\
20.95 \\
16.46 \\
10.01 \\
16.94 \\
8.79 \\
12.74 \\
7.36 \\
33.46 \\
20.81\end{array}$ & \begin{tabular}{|r|}
9.98 \\
15.96 \\
6.70 \\
13.48 \\
16.04 \\
3.08 \\
5.72 \\
10.44 \\
21.64 \\
8.18
\end{tabular} & \begin{tabular}{|r|}
20.39 \\
26.44 \\
25.73 \\
13.55 \\
20.58 \\
20.84 \\
16.99 \\
9.03 \\
42.99 \\
32.50
\end{tabular} & \begin{tabular}{|l}
22.81 \\
31.87 \\
29.82 \\
30.96 \\
30.64 \\
23.39 \\
18.40 \\
29.88 \\
45.86 \\
33.30
\end{tabular} \\
\hline $\begin{array}{l}\text { Mich. } \\
\text { Minn. } \\
\text { Miss. } \\
\text { Mo. } \\
\text { Mont. } \\
\text { Neb. } \\
\text { Nev. } \\
\text { N. C. } \\
\text { N. D. } \\
\text { N. H. }\end{array}$ & $\begin{array}{l}48.50 \\
33.73 \\
42.43 \\
46.15 \\
44.39 \\
45.83 \\
50.59 \\
35.51 \\
41.49 \\
53.69\end{array}$ & $\begin{array}{l}36.38 \\
15.85 \\
17.61 \\
24.00 \\
31.67 \\
18.79 \\
35.41 \\
10.51 \\
19.92 \\
31.60\end{array}$ & $\begin{array}{l}75.00 \\
47.00 \\
41.50 \\
52.00 \\
71.35 \\
41.00 \\
70.00 \\
29.60 \\
48.00 \\
58.85\end{array}$ & $\begin{array}{r}14.01 \\
12.86 \\
1.04 \\
4.50 \\
12.65 \\
8.82 \\
13.27 \\
.52 \\
2.91 \\
17.62\end{array}$ & $\begin{array}{l}70.95 \\
71.39 \\
89.62 \\
60.44 \\
73.26 \\
68.93 \\
45.45 \\
84.66 \\
46.72 \\
75.59\end{array}$ & $\begin{array}{r}18.97 \\
32.96 \\
5.52 \\
14.13 \\
34.36 \\
23.10 \\
31.88 \\
3.52 \\
30.30 \\
20.33\end{array}$ & $\begin{array}{r}9.20 \\
11.12 \\
2.34 \\
6.52 \\
15.25 \\
10.59 \\
16.13 \\
1.25 \\
12.57 \\
10.92\end{array}$ & $\begin{array}{r}13.94 \\
19.72 \\
6.31 \\
16.42 \\
24.31 \\
13.32 \\
26.81 \\
4.22 \\
13.18 \\
11.30\end{array}$ & $\left|\begin{array}{r}14.36 \\
26.12 \\
1.42 \\
10.20 \\
25.96 \\
17.10 \\
10.12 \\
1.38 \\
23.74 \\
15.86\end{array}\right|$ & $\left|\begin{array}{r|}17.32 \\
23.82 \\
11.00 \\
20.98 \\
30.25 \\
16.78 \\
45.08 \\
6.78 \\
16.04 \\
13.78\end{array}\right|$ & \begin{tabular}{|l|l}
31.86 \\
29.45 \\
21.88 \\
25.54 \\
36.34 \\
26.43 \\
34.43 \\
34.47 \\
17.80 \\
25.48 \\
30.95
\end{tabular} \\
\hline $\begin{array}{l}\text { N. J. } \\
\text { N. M. } \\
\text { N. Y. } \\
\text { Ohio } \\
\text { Ore. } \\
\text { Penn. } \\
\text { R. I. } \\
\text { S. C. } \\
\text { S. D. } \\
\text { Tenn. }\end{array}$ & $\begin{array}{l}45.26 \\
27.84 \\
51.48 \\
52.71 \\
51.23 \\
57.10 \\
50.29 \\
34.57 \\
50.18 \\
53.50\end{array}$ & $\begin{array}{r}43.45 \\
8.77 \\
48.01 \\
40.06 \\
30.28 \\
42.14 \\
47.27 \\
12.03 \\
24.09 \\
23.00\end{array}$ & $\begin{array}{l}96.00 \\
31.50 \\
93.25 \\
76.00 \\
59.10 \\
73.80 \\
94.00 \\
34.80 \\
48.00 \\
43.00\end{array}$ & $\begin{array}{r}8.89 \\
12.62 \\
11.14 \\
4.20 \\
4.90 \\
16.02 \\
1.06 \\
2.16 \\
.90\end{array}$ & $\begin{array}{l}89.93 \\
67.71 \\
63.78 \\
76.48 \\
66.15 \\
24.76 \\
57.47 \\
60.47\end{array}$ & $\begin{array}{r}23.86 \\
7.25 \\
27.28 \\
19.30 \\
18.60 \\
18.93 \\
26.10 \\
3.38 \\
24.82 \\
4.72\end{array}$ & $\begin{array}{r}11.28 \\
2.02 \\
14.05 \\
10.17 \\
9.53 \\
10.81 \\
13.13 \\
1.08 \\
12.46 \\
2.52\end{array}$ & $\begin{array}{r}31.01 \\
7.68 \\
23.06 \\
17.56 \\
13.09 \\
22.00 \\
26.76 \\
4.40 \\
10.77 \\
7.73\end{array}$ & $\begin{array}{r}15.58 \\
3.50 \\
21.80 \\
13.20 \\
10.48 \\
17.54 \\
18.66 \\
.82 \\
16.16 \\
1.92\end{array}$ & $\begin{array}{r}41.77 \\
11.65 \\
27.69 \\
23.11 \\
18.80 \\
23.61 \\
34.37 \\
7.73 \\
14.52 \\
12.32\end{array}$ & $\begin{array}{l}\mathbf{3 7 . 4 9} \\
\mathbf{1 0 . 0 2} \\
\mathbf{4 0 . 9 2} \\
\mathbf{3 3 . 0 9} \\
\mathbf{2 7 . 9 1} \\
\mathbf{3 4 . 7 0} \\
\mathbf{3 9 . 2 7} \\
\mathbf{1 2 . 4 6} \\
\mathbf{2 6 . 0 6} \\
\mathbf{2 1 . 0 1}\end{array}$ \\
\hline $\begin{array}{l}\text { Texas } \\
\text { Utah } \\
\text { Va. } \\
\text { Vt. } \\
\text { Wash. } \\
\text { Wis. } \\
\text { W. Va. } \\
\text { Wyo. }\end{array}$ & $\begin{array}{l}37.20 \\
31.54 \\
35.05 \\
56.02 \\
46.62 \\
39.77 \\
47.45 \\
33.20\end{array}$ & $\begin{array}{l}18.60 \\
20.97 \\
19.77 \\
38.09 \\
22.66 \\
32.81 \\
23.01 \\
19.75\end{array}$ & $\begin{array}{l}50.00 \\
66.50 \\
56.40 \\
68.00 \\
48.60 \\
82.50 \\
48.50 \\
59.50\end{array}$ & $\begin{array}{r}3.67 \\
.50 \\
3.11 \\
16.91 \\
2.60 \\
12.83 \\
1.71 \\
8.30\end{array}$ & $\begin{array}{l}71.83 \\
75.00 \\
69.74 \\
74.54 \\
73.91 \\
72.32 \\
55.26 \\
65.28\end{array}$ & $\begin{array}{r}10.89 \\
18.83 \\
8.10 \\
15.50 \\
25.93 \\
18.96 \\
9.85 \\
32.55\end{array}$ & $\begin{array}{r}4.05 \\
5.94 \\
2.84 \\
8.68 \\
12.09 \\
7.54 \\
4.67 \\
10.81\end{array}$ & $\begin{array}{r}12.17 \\
24.19 \\
9.90 \\
6.74 \\
24.80 \\
13.16 \\
9.10 \\
22.52\end{array}$ & $\begin{array}{r}3.76 \\
19.16 \\
2.96 \\
7.50 \\
27.90 \\
12.30 \\
5.68 \\
18.60\end{array}$ & \begin{tabular}{l|l} 
\\
8 \\
0 \\
0 \\
17.72 .95 \\
32.17
\end{tabular} & \begin{tabular}{l|l}
5 & 23.23 \\
7 & 28.64 \\
5 & 22.25 \\
1 & 30.22 \\
1 & 30.80 \\
7 & 30.99 \\
5 & 21.82 \\
70.27
\end{tabular} \\
\hline
\end{tabular}


Standing of the States IN 1900 Table 4 gives the 10 items in the index numbers for the school year ended in 1900. During the 10 years that had intervened since the preceding decade year there was a general increase in the figures for school attendance and expenditures which brought about an average increase in the index numbers of about five points. In this table, Oklahoma appears for the first time. The leading state is still Massachusetts with an index number of 49.52, followed by New York, California, Connecticut, and Rhode Island in that order. The lowest number is 17.51 for North Carolina. The difference between the highest state number and the lowest number in 1890 was approximately 35, but in 1900 the difference had been somewhat reduced and was 32. The average increase in the index numbers for all states was about four points.

It is interesting to note that during these decades, when Massachusetts was clearly the leading state in this composite measurement, the Commonwealth not only made a better showing than the other states in the purely educational items of the index, but also spent distinctly more per child for the support of education. 
TABLE 4.-COMPONENTS AND INDEX NUMBERS OF STATES. 1900

\begin{tabular}{|c|c|c|c|c|c|c|c|c|c|c|c|}
\hline State & 1 & 2 & 3 & 4 & 5 & 6 & 7 & 8 & 9 & 10 & Index \\
\hline $\begin{array}{l}\text { Ala. } \\
\text { Ariz. } \\
\text { Ark. } \\
\text { Cal. } \\
\text { Colo. } \\
\text { Conn. } \\
\text { D. C. } \\
\text { Del. } \\
\text { Fla. } \\
\text { Ga. }\end{array}$ & \begin{tabular}{|l}
45.61 \\
32.58 \\
41.75 \\
56.03 \\
61.69 \\
52.18 \\
50.12 \\
46.92 \\
42.71 \\
37.90
\end{tabular} & $\begin{array}{l}17.85 \\
20.35 \\
16.20 \\
46.55 \\
46.25 \\
49.30 \\
44.70 \\
37.65 \\
21.60 \\
21.75\end{array}$ & $\begin{array}{l}39.15 \\
62.50 \\
38.75 \\
83.10 \\
74.90 \\
94.50 \\
90.50 \\
80.00 \\
46.50 \\
56.00\end{array}$ & $\begin{array}{r}3.85 \\
5.07 \\
4.95 \\
19.18 \\
24.19 \\
21.79 \\
29.03 \\
13.91 \\
6.01 \\
5.88\end{array}$ & $\begin{array}{l}63.19 \\
49.57 \\
73.99 \\
66.27 \\
65.41 \\
76.70 \\
61.99 \\
61.85 \\
58.88 \\
60.44\end{array}$ & $\begin{array}{r}3.46 \\
29.45 \\
7.01 \\
35.00 \\
38.12 \\
28.58 \\
34.63 \\
13.99 \\
10.21 \\
6.64\end{array}$ & \begin{tabular}{|r|}
1.58 \\
9.59 \\
2.93 \\
19.61 \\
23.52 \\
14.92 \\
17.36 \\
5.69 \\
4.36 \\
2.52
\end{tabular} & $\begin{array}{r}7.83 \\
31.30 \\
8.20 \\
37.86 \\
32.36 \\
31.94 \\
41.74 \\
13.64 \\
11.64 \\
8.15\end{array}$ & $\begin{array}{r}.71 \\
21.72 \\
1.64 \\
15.38 \\
11.62 \\
20.94 \\
21.98 \\
5.08 \\
5.66 \\
1.12\end{array}$ & $\begin{array}{l}11.70 \\
39.51 \\
14.47 \\
59.07 \\
37.88 \\
40.46 \\
57.00 \\
22.32 \\
16.89 \\
14.93\end{array}$ & $\begin{array}{l}19.50 \\
30.17 \\
20.99 \\
43.80 \\
41.59 \\
43.13 \\
44.90 \\
30.10 \\
22.45 \\
21.54\end{array}$ \\
\hline $\begin{array}{l}\text { Idaho } \\
\text { Ill. } \\
\text { Ind. } \\
\text { Iowa } \\
\text { Kans. } \\
\text { Ky. } \\
\text { La. } \\
\text { Maine } \\
\text { Mass. } \\
\text { Md. }\end{array}$ & $\begin{array}{l}50.16 \\
54.13 \\
57.79 \\
55.00 \\
56.35 \\
45.84 \\
31.11 \\
57.17 \\
57.08 \\
38.42\end{array}$ & $\begin{array}{l}26.60 \\
43.40 \\
43.90 \\
43.45 \\
35.55 \\
26.45 \\
18.65 \\
40.30 \\
53.70 \\
36.10\end{array}$ & $\begin{array}{l}53.00 \\
76.00 \\
76.00 \\
79.00 \\
63.10 \\
57.70 \\
60.00 \\
70.50 \\
94.50 \\
94.00\end{array}$ & $\begin{array}{r}6.64 \\
15.23 \\
18.45 \\
23.89 \\
17.09 \\
5.36 \\
4.54 \\
26.86 \\
29.43 \\
8.94\end{array}$ & $\begin{array}{l}80.00 \\
64.40 \\
73.43 \\
68.25 \\
64.91 \\
72.14 \\
58.10 \\
77.79 \\
77.70 \\
76.92\end{array}$ & $\begin{array}{r}18.22 \\
24.07 \\
19.28 \\
21.89 \\
17.66 \\
8.58 \\
7.76 \\
17.53 \\
37.76 \\
21.95\end{array}$ & \begin{tabular}{|r}
9.14 \\
13.03 \\
11.02 \\
12.04 \\
9.95 \\
3.93 \\
2.41 \\
10.02 \\
21.55 \\
8.43
\end{tabular} & $\begin{array}{l}15.73 \\
28.12 \\
21.84 \\
11.59 \\
16.74 \\
11.09 \\
11.38 \\
11.07 \\
42.42 \\
23.67\end{array}$ & $\begin{array}{r}11.66 \\
16.78 \\
15.94 \\
14.04 \\
11.08 \\
3.28 \\
2.62 \\
9.90 \\
30.88 \\
11.74\end{array}$ & $\begin{array}{l}21.38 \\
36.63 \\
25.61 \\
15.74 \\
22.97 \\
17.95 \\
18.93 \\
15.89 \\
50.15 \\
34.67\end{array}$ & $\begin{array}{l}29.25 \\
37.18 \\
36.33 \\
34.49 \\
31.54 \\
25.23 \\
21.55 \\
33.70 \\
49.52 \\
35.49\end{array}$ \\
\hline $\begin{array}{l}\text { Mo. } \\
\text { Mont. } \\
\text { Neb. } \\
\text { Nev. } \\
\text { N. C. } \\
\text { N. D. } \\
\text { N. H. }\end{array}$ & $\begin{array}{l}52.87 \\
47.99 \\
36.08 \\
47.60 \\
54.84 \\
56.52 \\
50.73 \\
30.91 \\
49.90 \\
52.51\end{array}$ & $\begin{array}{l}42.75 \\
36.15 \\
18.95 \\
34.40 \\
38.35 \\
46.00 \\
39.05 \\
10.95 \\
38.95 \\
35.50\end{array}$ & $\begin{array}{l}80.90 \\
84.50 \\
52.55 \\
72.00 \\
70.00 \\
67.50 \\
77.00 \\
35.40 \\
77.85 \\
67.65\end{array}$ & $\begin{array}{r}24.70 \\
15.19 \\
6.03 \\
13.44 \\
20.35 \\
25.08 \\
27.52 \\
1.37 \\
7.78 \\
23.28\end{array}$ & $\begin{array}{l}72.86 \\
68.86 \\
66.47 \\
66.19 \\
64.65 \\
66.12 \\
61.42 \\
75.28 \\
64.24 \\
76.21\end{array}$ & $\begin{array}{r}18.68 \\
23.15 \\
6.48 \\
16.99 \\
35.44 \\
24.22 \\
47.81 \\
4.34 \\
33.08 \\
22.02\end{array}$ & \begin{tabular}{|r|}
9.88 \\
11.11 \\
2.34 \\
8.09 \\
19.43 \\
13.68 \\
24.26 \\
1.39 \\
16.54 \\
11.56
\end{tabular} & $\begin{array}{r}17.51 \\
22.15 \\
6.68 \\
20.10 \\
29.31 \\
19.39 \\
28.89 \\
5.25 \\
14.71 \\
14.75\end{array}$ & $\begin{array}{r}12.72 \\
14.70 \\
1.80 \\
13.62 \\
23.56 \\
19.42 \\
28.34 \\
1.58 \\
28.62 \\
15.64\end{array}$ & $\begin{array}{r}23.09 \\
30.24 \\
11.50 \\
24.10 \\
39.14 \\
23.23 \\
40.65 \\
8.59 \\
16.68 \\
19.02\end{array}$ & $\begin{array}{l}35.60 \\
35.41 \\
20.89 \\
31.65 \\
39.51 \\
36.11 \\
42.37 \\
17.51 \\
34.83 \\
33.82\end{array}$ \\
\hline $\begin{array}{l}\text { N. J. } \\
\text { N. M. } \\
\text { N. Y. } \\
\text { Ohio } \\
\text { Okla. } \\
\text { Ore. } \\
\text { Penn. } \\
\text { R. I. } \\
\text { S. C. } \\
\text { S. D. }\end{array}$ & $\begin{array}{l}42.38 \\
40.92 \\
48.01 \\
52.25 \\
53.01 \\
57.77 \\
48.58 \\
45.05 \\
40.43 \\
57.82\end{array}$ & $\begin{array}{l}39.20 \\
19.75 \\
44.65 \\
43.10 \\
25.25 \\
33.70 \\
40.45 \\
42.70 \\
17.90 \\
46.65\end{array}$ & $\begin{array}{l}92.50 \\
48.30 \\
87.50 \\
82.50 \\
47.65 \\
58.30 \\
83.35 \\
93.50 \\
44.20 \\
64.55\end{array}$ & $\begin{array}{r}16.64 \\
3.25 \\
21.82 \\
22.25 \\
1.58 \\
8.92 \\
11.37 \\
22.46 \\
5.96 \\
11.55\end{array}$ & $\begin{array}{l}60.67 \\
69.93 \\
87.01 \\
76.09 \\
53.42 \\
63.34 \\
62.34 \\
74.77 \\
73.45 \\
73.77\end{array}$ & $\begin{array}{r}30.26 \\
15.31 \\
38.97 \\
21.63 \\
10.77 \\
24.75 \\
25.12 \\
34.09 \\
4.44 \\
23.51\end{array}$ & $\begin{array}{r}12.82 \\
6.26 \\
18.71 \\
11.30 \\
5.71 \\
14.30 \\
12.21 \\
15.36 \\
1.80 \\
13.59\end{array}$ & $\begin{array}{r}38.26 \\
14.82 \\
39.96 \\
21.36 \\
12.20 \\
17.76 \\
30.45 \\
34.22 \\
6.70 \\
13.87\end{array}$ & $\begin{array}{r}25.10 \\
10.32 \\
33.12 \\
13.78 \\
9.42 \\
21.62 \\
24.02 \\
24.86 \\
1.48 \\
16.84\end{array}$ & $\begin{array}{l}44.80 \\
19.65 \\
45.95 \\
29.11 \\
13.72 \\
20.00 \\
31.77 \\
43.49 \\
11.15 \\
17.81\end{array}$ & $\begin{array}{l}40.26 \\
24.86 \\
46.57 \\
37.34 \\
23.27 \\
32.04 \\
36.97 \\
43.05 \\
20.75 \\
33.99\end{array}$ \\
\hline $\begin{array}{l}\text { Tenn. } \\
\text { Texas } \\
\text { Utah } \\
\text { Va. } \\
\text { Vt. } \\
\text { Wash. } \\
\text { Wis. } \\
\text { W.Va. } \\
\text { Wyo. }\end{array}$ & $\begin{array}{l}48.95 \\
36.80 \\
56.34 \\
32.44 \\
55.50 \\
59.05 \\
50.11 \\
46.92 \\
51.47\end{array}$ & $\begin{array}{l}23.50 \\
19.90 \\
41.25 \\
19.30 \\
43.35 \\
43.70 \\
40.10 \\
24.85 \\
28.30\end{array}$ & $\begin{array}{l}48.00 \\
54.10 \\
75.50 \\
59.50 \\
78.00 \\
74.00 \\
80.00 \\
53.00 \\
55.00\end{array}$ & $\begin{array}{r}4.80 \\
11.37 \\
6.61 \\
6.40 \\
21.94 \\
16.18 \\
19.97 \\
3.88 \\
10.54\end{array}$ & \begin{tabular}{|l|}
66.68 \\
68.67 \\
78.69 \\
58.38 \\
75.77 \\
62.05 \\
73.65 \\
51.55 \\
76.73
\end{tabular} & $\begin{array}{r}5.17 \\
11.35 \\
21.21 \\
9.70 \\
22.85 \\
27.98 \\
17.73 \\
21.27 \\
24.95\end{array}$ & \begin{tabular}{|r|}
2.53 \\
4.18 \\
11.95 \\
3.15 \\
12.68 \\
16.52 \\
8.88 \\
9.97 \\
12.84
\end{tabular} & $\begin{array}{r}7.94 \\
12.40 \\
30.52 \\
9.30 \\
11.96 \\
22.53 \\
17.53 \\
18.66 \\
18.54\end{array}$ & $\mid \begin{array}{r}2.04 \\
2.42 \\
18.32 \\
4.60 \\
17.68 \\
22.28 \\
11.18 \\
26.50 \\
14.42\end{array}$ & $\left\{\begin{array}{l}12.72 \\
22.14 \\
34.66 \\
14.19 \\
14.67 \\
27.12 \\
23.99 \\
14.09 \\
26.37\end{array}\right.$ & $\begin{array}{l}22.23 \\
24.43 \\
37.51 \\
21.69 \\
35.44 \\
37.14 \\
34.31 \\
27.07 \\
31.91\end{array}$ \\
\hline
\end{tabular}


Standing of the States in 1910

Table 5 gives the 10 items in the index number for the 48 states and the District of Columbia for the school year ended in 1910. In this case the leading state is Washington which had made striking progress during the two previous decades and appears, in 1910, with an index number of 61.21. It is followed by California, Massachusetts, Nevada, and New Jersey, and the lowest number is $\mathbf{2 4 . 8 7}$ for South Carolina. In this instance the difference between the highest record and the lowest is approximately 36 , which is somewhat greater than the differences in the two previous decade years. The average gain in the index numbers was about nine points as compared with four during the previous decade.

It is noteworthy that California always appears among the leaders in these comparisons. The state consistently shows a high record in each of the years compared and if all the comparisons that have been made are taken into consideration it is found that California has not only the most consistently high record among the states, but also the highest average record of all. 
TABLE 5.-COMPONENTS AND INDEX NUMBERS OF STATES. 1910

\begin{tabular}{|c|c|c|c|c|c|c|c|c|c|c|c|}
\hline State & 1 & 2 & 3 & 4 & 5 & 6 & 7 & 8 & 9 & 10 & ndex \\
\hline $\begin{array}{l}\text { a. } \\
\text { iz. } \\
\text { k. } \\
\text { l. } \\
\text { lo. } \\
\text { onn. } \\
\text { C. } \\
\text { l. } \\
\text { a. } \\
\text { t. }\end{array}$ & $\begin{array}{l}38.98 \\
40.97 \\
47.94 \\
53.09 \\
55.78 \\
57.66 \\
62.12 \\
42.08 \\
54.83 \\
41.71\end{array}$ & $\begin{array}{l}22.90 \\
27.80 \\
25.55 \\
48.35 \\
43.55 \\
53.50 \\
56.35 \\
36.35 \\
31.65 \\
31.00\end{array}$ & $\begin{array}{l}58.65 \\
67.75 \\
53.25 \\
91.00 \\
78.00 \\
92.35 \\
90.60 \\
86.25 \\
53.00 \\
72.20\end{array}$ & $\begin{array}{r}9.02 \\
16.95 \\
7.75 \\
36.81 \\
34.13 \\
26.92 \\
34.74 \\
22.95 \\
9.49 \\
12.48\end{array}$ & $\begin{array}{l}72.52 \\
84.25 \\
72.59 \\
80.45 \\
75.24 \\
80.62 \\
74.42 \\
78.12 \\
67.23 \\
73.04\end{array}$ & $\begin{array}{l}11.04 \\
49.81 \\
12.49 \\
63.52 \\
48.47 \\
37.02 \\
60.05 \\
26.81 \\
17.07 \\
12.76\end{array}$ & \begin{tabular}{|r|}
4.30 \\
20.40 \\
5.99 \\
33.73 \\
27.03 \\
21.35 \\
37.30 \\
11.28 \\
9.36 \\
5.33
\end{tabular} & $\begin{array}{l}13.82 \\
48.99 \\
13.95 \\
66.74 \\
41.76 \\
43.03 \\
18.45 \\
25.38 \\
18.41 \\
14.58\end{array}$ & $\begin{array}{r}11.86 \\
30.42 \\
3.75 \\
54.27 \\
34.87 \\
33.80 \\
54.62 \\
17.13 \\
12.78 \\
7.28\end{array}$ & \begin{tabular}{|l|}
26.13 \\
68.06 \\
23.70 \\
76.45 \\
53.47 \\
46.76 \\
74.62 \\
34.53 \\
23.04 \\
20.84
\end{tabular} & $\begin{array}{l}60.44 \\
49.23 \\
49.31 \\
56.33\end{array}$ \\
\hline $\begin{array}{l}\text { bo } \\
\text { as } \\
\text { as. } \\
\text { ine } \\
\text { es. }\end{array}$ & $\begin{array}{l}56.12 \\
55.26 \\
61.26 \\
56.81 \\
58.01 \\
44.55 \\
33.93 \\
62.10 \\
60.62 \\
40.33\end{array}$ & $\begin{array}{l}38.35 \\
47.55 \\
45.05 \\
48.85 \\
47.45 \\
27.85 \\
23.05 \\
49.40 \\
56.90 \\
37.35\end{array}$ & $\begin{array}{l}68.50 \\
85.50 \\
73.50 \\
86.00 \\
81.75 \\
62.50 \\
67.80 \\
79.50 \\
93.00 \\
92.50\end{array}$ & $\begin{array}{r}18.04 \\
24.32 \\
31.22 \\
32.93 \\
27.06 \\
9.21 \\
8.01 \\
31.04 \\
37.03 \\
16.74\end{array}$ & \begin{tabular}{|l|}
74.77 \\
77.88 \\
83.87 \\
72.29 \\
69.35 \\
72.99 \\
64.73 \\
72.70 \\
86.21 \\
76.58
\end{tabular} & $\begin{array}{l}42.52 \\
43.70 \\
35.43 \\
35.44 \\
33.69 \\
17.92 \\
23.27 \\
27.43 \\
45.35 \\
26.01\end{array}$ & $\begin{array}{r}23.86 \\
24.14 \\
21.71 \\
13.14 \\
19.54 \\
7.98 \\
7.90 \\
17.04 \\
27.69 \\
10.49\end{array}$ & & $\begin{array}{l}37.12 \\
43.00 \\
27.97 \\
24.60 \\
27.73 \\
12.07 \\
18.02 \\
20.84 \\
38.44 \\
13.03\end{array}$ & $\begin{array}{l}45.77 \\
49.03 \\
43.54 \\
25.17 \\
35.72 \\
28.12 \\
34.55 \\
20.34 \\
63.10 \\
42.96\end{array}$ & \\
\hline $\begin{array}{l}\text { Mich. } \\
\text { Minn. } \\
\text { Miss. } \\
\text { Mo. } \\
\text { Mont. } \\
\text { Neb. } \\
\text { Nev. } \\
\text { N. C. } \\
\text { N. D. } \\
\text { N. H. }\end{array}$ & $\begin{array}{l}58.45 \\
57.11 \\
42.77 \\
50.48 \\
49.26 \\
53.25 \\
42.43 \\
48.02 \\
53.87 \\
53.87\end{array}$ & $\begin{array}{l}49 . \\
42 . \\
28 . \\
39 . \\
45 . \\
46 . \\
30 . \\
24 . \\
39 . \\
44 .\end{array}$ & & $\begin{array}{r}27.05 \\
22.96 \\
8.85 \\
20.11 \\
21.77 \\
30.59 \\
32.26 \\
7.70 \\
14.69 \\
32.61\end{array}$ & & $\begin{array}{r}32.92 \\
39.38 \\
10.43 \\
26.64 \\
63.76 \\
39.01 \\
83.66 \\
9.17 \\
50.46 \\
33.02\end{array}$ & $\begin{array}{r}19.24 \\
22.46 \\
4.46 \\
13.45 \\
31.39 \\
20.77 \\
35.51 \\
4.40 \\
27.19 \\
17.78\end{array}$ & & $\begin{array}{r}36 . \\
4 . \\
20 . \\
57 . \\
30 . \\
105 . \\
4 . \\
45 . \\
26 .\end{array}$ & & \\
\hline $\begin{array}{l}\text { N. M. } \\
\text { N. Y. } \\
\text { Ohio } \\
\text { Okla. } \\
\text { Ore. } \\
\text { Penn. } \\
\text { R. I. } \\
\text { S. C. } \\
\text { S. D. }\end{array}$ & $\begin{array}{l}51.94 \\
43.69 \\
54.31 \\
60.29 \\
53.72 \\
61.57 \\
52.94 \\
51.02 \\
46.52 \\
47.26\end{array}$ & $\begin{array}{l}47 . \\
21 . \\
51 . \\
51 . \\
32 . \\
42 . \\
44 . \\
49 . \\
24 . \\
39 .\end{array}$ & & $\begin{array}{r}20.87 \\
10.78 \\
27.56 \\
28.97 \\
9.56 \\
25.81 \\
19.48 \\
32.77 \\
6.86 \\
21.51\end{array}$ & & $\mid \begin{array}{r}52.65 \\
21.22 \\
46.22 \\
39.32 \\
24.18 \\
44.85 \\
39.95 \\
40.46 \\
8.00 \\
47.32\end{array}$ & $\begin{array}{r}27.34 \\
9.27 \\
25.09 \\
23.70 \\
12.99 \\
27.62 \\
21.13 \\
20.64 \\
3.72 \\
22.60\end{array}$ & & & $\begin{array}{l}29.03 \\
67.77 \\
43.70 \\
33.99 \\
43.03 \\
46.15 \\
50.64 \\
17.64 \\
27.45\end{array}$ & $\begin{array}{l}51.8 \\
48.6 \\
35.9 \\
47.8 \\
47.2 \\
50.8 \\
24.8 \\
42.5\end{array}$ \\
\hline $\begin{array}{l}\text { an. } \\
\text { xas } \\
\text { ah } \\
\text { ish. } \\
\text { s. }_{\text {. }}\end{array}$ & $\begin{array}{l}52.21 \\
42.05 \\
56.89 \\
39.82 \\
66.55 \\
60.47 \\
46.23 \\
53.66 \\
54.17\end{array}$ & $\begin{array}{l}33.95 \\
27.50 \\
46.85 \\
27.90 \\
53.35 \\
52.25 \\
41.65 \\
35.90 \\
40.20\end{array}$ & $\begin{array}{l}65.00 \\
65.50 \\
82.40 \\
70.00 \\
80.00 \\
86.00 \\
90.00 \\
67.00\end{array}$ & $\begin{array}{r}7.78 \\
16.74 \\
15.59 \\
13.38\end{array}$ & $\begin{array}{l}67.67 \\
71.17 \\
87.55 \\
69.83 \\
71.99\end{array}$ & $\begin{array}{l}12.10 \\
21.62 \\
44.09 \\
16.99 \\
30.88 \\
67.22 \\
33.67 \\
21.56 \\
47.58\end{array}$ & $\begin{array}{r}6.32 \\
9.09 \\
25.09 \\
6.77 \\
20.55 \\
40.65 \\
15\end{array}$ & & $\begin{array}{r}7.66 \\
13.97 \\
47.66 \\
12.39 \\
28.51 \\
70.89 \\
25.41 \\
13.22 \\
36.91\end{array}$ & $\begin{array}{l}24.36 \\
32.03 \\
49.34 \\
22.36 \\
22.16\end{array}$ & $\begin{array}{l}50.92 \\
29.70 \\
42.11 \\
61.21 \\
43.23 \\
32.87 \\
42.59\end{array}$ \\
\hline
\end{tabular}




\section{Standing of the States in 1918}

Table 6 gives the 10 items in the index numbers for 1918. During the eight years intervening since the previous decade year many important changes took place. The leading state is Montana with an index number of 75.79, followed by California, Arizona, New Jersey, and Washington. The lowest record is that of South Carolina with an index number of 29.39. It is noteworthy that, while the difference between the highest index number and the lowest in the previous decade years was 35,32 , and 36 , it had by 1918 increased to more than 46 . The evidence indicates that, while the states with the lowest numbers had made relatively slight progress, those with the highest numbers had made marked progress. The average increase during the eight years of the period was about nine points.

During the period from 1910 to 1918 a number of striking changes took place. Iowa advanced from 30 th place to seventh place, and Michigan from 19th to 10th. In the same period Rhode Island fell from 10th place to 26 th. 
TABLE 6.-COMPONENTS AND INDEX NUMBERS OF STATES. 1918

\begin{tabular}{|c|c|c|c|c|c|c|c|c|c|c|c|}
\hline & 1 & 2 & 3 & 4 & 5 & 6 & 7 & 8 & 9 & 10 & Index \\
\hline $\begin{array}{l}\text { la. } \\
\text { riz. } \\
\text { rk. } \\
\text { al. } \\
\text { olo. } \\
\text { onn. } \\
\text { Z. } \\
\text { c. C. } \\
\text { la. }\end{array}$ & $\begin{array}{l}44.02 \\
62.04 \\
53.29 \\
70.85 \\
58.12 \\
64.71 \\
25.73 \\
64.19 \\
45.87 \\
50.93\end{array}$ & $\begin{array}{l}27.75 \\
50.26 \\
31.95 \\
61.10 \\
48.80 \\
58.70 \\
23.03 \\
55.50 \\
37.60 \\
33.10\end{array}$ & $\begin{array}{l}62.00 \\
81.00 \\
60.00 \\
86.00 \\
84.00 \\
90.50 \\
89.50 \\
86.50 \\
82.00 \\
65.00\end{array}$ & $\begin{array}{l}16.27 \\
27.34 \\
11.73 \\
58.79 \\
40.16 \\
35.13 \\
30.09 \\
43.44 \\
31.28 \\
17.83\end{array}$ & $\begin{array}{l}70.11 \\
81.77 \\
68.58 \\
77.82 \\
72.46 \\
79.30 \\
78.72 \\
76.05 \\
72.56 \\
64.23\end{array}$ & $\begin{array}{l}17.75 \\
85.43 \\
16.65 \\
79.41 \\
70.53 \\
55.89 \\
72.72 \\
66.55 \\
35.59 \\
34.04\end{array}$ & $\begin{array}{r}7.98 \\
53.00 \\
8.87 \\
56.26 \\
41.00 \\
36.16 \\
28.26 \\
42.72 \\
16.32 \\
17.34\end{array}$ & $\begin{array}{l}20.13 \\
69.06 \\
17.34 \\
72.04 \\
54.31 \\
60.52 \\
66.08 \\
69.93 \\
34.24 \\
30.60\end{array}$ & $\begin{array}{r}10.16 \\
72.26 \\
7.74 \\
61.76 \\
62.48 \\
50.85 \\
52.60 \\
46.51 \\
22.56 \\
33.45\end{array}$ & $\begin{array}{l}28.73 \\
79.70 \\
26.60 \\
88.06 \\
60.49 \\
65.97 \\
84.37 \\
90.98 \\
46.77 \\
31.13\end{array}$ & $\begin{array}{l}30.58 \\
66.19 \\
30.28 \\
71.21 \\
59.23 \\
59.77 \\
55.11 \\
64.24 \\
42.48 \\
37.77\end{array}$ \\
\hline $\begin{array}{l}\text { awaii } \\
\text { aho } \\
\text { d. } \\
\text { wa } \\
\text { ans. } \\
\text { y. } \\
\text { aine }\end{array}$ & $\begin{array}{l}49.12 \\
72.74 \\
61.25 \\
61.88 \\
73.37 \\
67.85 \\
58.16 \\
44.51 \\
39.54 \\
63.54\end{array}$ & & $\begin{array}{l}69.00 \\
92.00 \\
75.00 \\
79.50 \\
77.50 \\
90.00 \\
86.00 \\
75.00 \\
68.00 \\
84.50\end{array}$ & $\begin{array}{r}15.46 \\
6.94 \\
38.44 \\
32.68 \\
37.87 \\
42.00 \\
51.79 \\
19.41 \\
19.64 \\
47.21\end{array}$ & $\begin{array}{l}73.66 \\
51.86 \\
71.78 \\
78.62 \\
82.94 \\
68.87 \\
68.44 \\
67.13 \\
59.24 \\
73.29\end{array}$ & $\begin{array}{l}16.53 \\
52.04 \\
69.41 \\
53.53 \\
52.72 \\
74.03 \\
59.34 \\
26.53 \\
25.79 \\
38.97\end{array}$ & $\begin{array}{r}8.12 \\
37.85 \\
42.52 \\
33.12 \\
38.68 \\
50.23 \\
34.51 \\
11.81 \\
10.20 \\
24.75\end{array}$ & & $\begin{array}{r}7.18 \\
48.16 \\
68.15 \\
49.58 \\
58.69 \\
79.24 \\
57.42 \\
17.87 \\
17.42 \\
31.65\end{array}$ & $\begin{array}{l}32.31 \\
73.62 \\
57.11 \\
66.95 \\
51.38 \\
41.04 \\
43.71 \\
30.89 \\
40.95 \\
30.40\end{array}$ & $\begin{array}{l}32.60 \\
57.07 \\
58.57 \\
\mathbf{5 6 . 7 5} \\
\mathbf{5 8 . 8 0} \\
\mathbf{6 1 . 8 5} \\
\mathbf{5 5 . 1 6} \\
\mathbf{3 4 . 9 8} \\
\mathbf{3 3 . 8 6} \\
\mathbf{4 7 . 3 6}\end{array}$ \\
\hline $\begin{array}{l}\text { lass. } \\
\text { di. } \\
\text { lich. } \\
\text { linn. } \\
\text { liss. } \\
\text { lo. } \\
\text { lont. } \\
\text { eb. } \\
\text { ev. } \\
\text { c. }\end{array}$ & $\begin{array}{l}60.58 \\
44.54 \\
67.87 \\
58.85 \\
53.11 \\
56.18 \\
86.24 \\
60.92 \\
55.39 \\
52.35\end{array}$ & $\begin{array}{l}52.20 \\
37.95 \\
58.35 \\
49.70 \\
36.70 \\
46.45 \\
65.70 \\
50.30 \\
47.40 \\
32.20\end{array}$ & $\begin{array}{l}85.00 \\
86.00 \\
84.50 \\
69.00 \\
82.50 \\
76.00 \\
82.50 \\
85.50 \\
61.50\end{array}$ & $\begin{array}{l}46.13 \\
25.14 \\
36.67 \\
38.60 \\
10.96 \\
33.62 \\
34.67 \\
40.61 \\
44.01 \\
12.91\end{array}$ & & \begin{tabular}{|r|}
59.99 \\
36.98 \\
61.39 \\
66.87 \\
12.32 \\
45.26 \\
103.08 \\
67.08 \\
76.95 \\
15.77
\end{tabular} & $\begin{array}{r}36.34 \\
16.48 \\
41.67 \\
39.36 \\
6.55 \\
25.43 \\
88.90 \\
40.87 \\
42.62 \\
8.26\end{array}$ & & \begin{tabular}{|r|}
52.23 \\
23.56 \\
65.84 \\
68.37 \\
2.52 \\
37.12 \\
115.23 \\
64.65 \\
56.22 \\
9.32
\end{tabular} & $\begin{array}{l}71.53 \\
59.04 \\
52.21 \\
54.24 \\
26.79 \\
54.42 \\
57.42 \\
48.66 \\
57.75 \\
25.00\end{array}$ & $\begin{array}{l}61.04 \\
43.22 \\
60.43 \\
58.43 \\
30.04 \\
49.64 \\
75.79 \\
57.14 \\
59.05 \\
30.59\end{array}$ \\
\hline $\begin{array}{l}\text { N. D. } \\
\text { N. H. } \\
\text { N. J. } \\
\text { N. M. } \\
\text { N. Y. } \\
\text { Ohio } \\
\text { Okla. } \\
\text { Ore. } \\
\text { Penn. } \\
\text { P. R. }\end{array}$ & & & & $\begin{array}{r}29.20 \\
47.66 \\
35.92 \\
17.78 \\
37.61 \\
37.16 \\
28.45 \\
41.15 \\
30.93 \\
7.25\end{array}$ & & & & & & & \\
\hline C. & $\begin{array}{l}48.8 \\
46 . \\
44.4 \\
58 . \\
54 . \\
65 . \\
48 . \\
58 . \\
53 . \\
50 . \\
53 .\end{array}$ & $\begin{array}{l}46 \\
45\end{array}$ & & $\begin{array}{l}34.48 \\
10.28 \\
39.72 \\
12.29 \\
23.49 \\
32.00 \\
24.49 \\
47.85 \\
52.88 \\
41.20 \\
22.03\end{array}$ & $\mid \begin{array}{l}81 \\
58 \\
62 \\
70 \\
69 \\
83 \\
62\end{array}$ & $\begin{array}{l}56.03 \\
19.41 \\
78.27 \\
18.97 \\
31.15 \\
62.29 \\
25.45 \\
50.99 \\
78.32 \\
49.53 \\
31.42 \\
65.46\end{array}$ & \begin{tabular}{r|r}
27.45 \\
8.96 \\
74.78 \\
711.05 \\
5 \\
17.03 \\
40.67 \\
12.24 \\
00
\end{tabular} & & $\begin{array}{l}52.29 \\
17.62 \\
73.79 \\
14.32 \\
26.47 \\
62.10 \\
18.11 \\
46.07 \\
63.28 \\
49.14 \\
20.57 \\
53.99\end{array}$ & $\begin{array}{l}61.34 \\
26.25 \\
42.20 \\
30.85 \\
40.59 \\
64.12 \\
32.07 \\
37.81 \\
78.02 \\
44.03 \\
34.80 \\
49.67\end{array}$ & 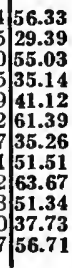 \\
\hline
\end{tabular}


Hawail, the Canal Zone, and Porto Rico Table 6 for 1918 gives, for the first time, figures for the school systems of three American possessions outside of continental United States. These are Hawaï, the Canal Zone, and Porto Rico. The 10 items for these systems have been gathered and the index numbers computed by the methods used for the 48 states. The index number for Hawaii is 57.07 , which gives that school system a rank of 23 among the 52 systems included in the Table. The number of Hawaii is just below those of Pennsylvania and Nebraska, and just above those of Illinois and Wyoming.

The index number for the Canal Zone is 55.11 with a rank of 28 th place from the top. It takes its position just below Rhode Island and Kansas and just above South Dakota and New Hampshire. The schools of Porto Rico have an index number of 35.79 and a rank of 42 among the 52 systems. Their number is just below those of Florida and West Virginia and just above the numbers for Virginia and Tennessee.

\section{Education in Porto Rico}

The data for Porto Rico are especially noteworthy. The index number indicates that 38 
the insular system has a rank above 10 of the state systems. This is particularly significant in view of the handicaps under which this marked progress has been made. The per capita wealth of the island is far less than that of the poorest American state. According to the census figures the per capita wealth of the United States in 1912 amounted to approximately $\$ 2,000$. That of the least wealthy states was something less than $\$ 800$, and the highest per capita wealth in any state was over $\$ 5,000$.

As contrasted with these figures the per capita wealth of Porto Rico is stated in 1919 as being $\$ 200$. These figures may be accepted as being reasonably trustworthy for comparative purposes. They indicate that the average American state is 10 times as wealthy as Porto Rico in proportion to its population, that the poorest state has nearly four times as much wealth per capita of population, and that the wealthiest one has something like 25 times as much wealth per inhabitant. Nevertheless, and in spite of these handicaps, Porto Rico keeps her public schools open for a greater number of days each year than most American states. In 1918 there were only 11 states having a longer average school 
term. Moreover, during the school year 1917-18 the island paid its teachers higher average salaries than 13 American states, and among these 13 are included practically all the Southern states and two of the New England states. The 'Porto Rican school system is supported from insular revenues without subventions from the Federal government. It has received no financial support from other than local sources, save that the island is permitted to retain the customs revenues from imports coming directly from foreign countries to its ports. It must be remembered, moreover, that the island has a very large colored population.

All of these facts tend to indicate that the educational effort of a state is dependent on its aspirations and ideals in even greater measure than on its financial resources. The handicap of restricted resources is relative rather than absolute.

In computing the expenditures for Porto Rico the disbursements of the local school boards have been included as well as those of the insular government. This procedure makes the data comparable with those for the states but employs figures which differ somewhat from those published by the Bureau of Education. 
It has not been possible to secure data for the school systems of Alaska and the Philippine Islands that could fairly be compared with those for the several states. Such figures as are available indicate that these systems are making most credible records with respect to the length of the school term, the payment of teachers, and certain of the other items. However, the reports are not sufficiently complete to make possible all the computations entering into the index number. 
Standing of the States in Four Decades The index numbers of the four preceding tables have been brought together and compared in Table 7. In the four columns of this table the index numbers for each decade year and 1918 are arranged in descending order from the highest to the lowest. The lines running from column to column connect the four records of each state and show whether the relative standing of the state, as shown by its index number, has moved upward or downward.

The complicated criss-crossing of the lines conveys the impression that the shifts in the index numbers have been varied and numerous. This impression is only partly warranted by the facts. In general the entire group of states has moved forward rather steadily. This progress has been greater on the part of northern states than among the southern ones, and more marked among western states than among eastern ones.

Since the position of each state is given for each year, even a few shifts in relative position occasion much crossing of lines. This is accentuated by the bringing in of the outlying possessions in the last year. 
1910

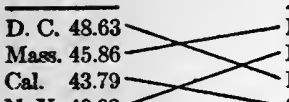

Mass. 49.52

N. Y. 40.92

Y. 46.57

R. I. 39.27 Cal. 43.80 Conn. 38.90 Colo. 37.83 N. J. 37.49 Conn. 43.13 Mont. 36.34 R. I. 43.05 Penn. 34.70 Nev. 34.47 Md. 33.30 Ohio 33.09 Ariz. 32.75 III. Mich. 31.86 Wis, 30.99 Iowa 30.96 N. H. 30.95 Wash. 30.80 Kans. 30.64 Wyo. 30.27
Vt. 30.22 Maine 29.88 Ind. $29.82 \times \times$ S. D. 33.99 Minn. 29.45 $/ \times$ N. H. 33.82 Del. $29.30 \times / X$ Maine 33.70 Utah 28.64

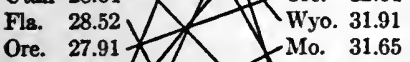
Neb. 26.43 S. D. 26.06 Mo. N. D. 25.48 Ky. $\quad 23.39$ Texas 23.23 Idaho 22.81 Va. $\quad 22.25$ Miss. 21.88 W.Va.21.82

Tenn. 21.01 Ark. 20.07 Kans. 31.54 Nev. 42.37 N. J. 40.26 I. A $18.40 \longrightarrow \mathrm{La}$ La. Ariz. 30.17 Del. 30.10

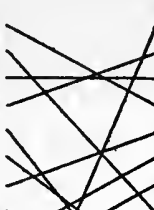
Wash.61.21 Mont.75.79 Utah $\begin{aligned} & 50.92 \\ & \text { R. I. }\end{aligned}{ }^{50.84}$ Dal. 60.44 
Table 8 is similar to Table 7. The difference is that in Table 7 the number following the name of the state was its index number, whereas in Table 8 the number precedes the name of the state and shows its rank in the order from highest to lowest.

Table 8 shows even more clearly than the preceding table the general upward trend of the western states and the comparative falling off of the eastern ones. During the 28 years from 1890 to 1918 the average rank of the states in the Eastern Division shows a falling off of eight places.

The average rank of these states in 1890 was 11, while 28 years later it had fallen to 19 . The states of the Western Division have moved upwards. In 1890 their average rank was 21, while in 1918 it had moved up to 13. New Jersey is the only state in the Eastern Division that has gained in relative rank during this period of 28 years. The western states, as a group, had gained eight points in average rank while the eastern states lost eight points. The states of the North Central Division show, in the same period, an average gain of three points, those in the South Atlantic Division an average loss of four points, and those in the South Central Division a loss of three points. 
TABLE 8.-RANKS OF STATES AS SHOWN BY INDEX NUMBERS

FOR FOUR PERIODS

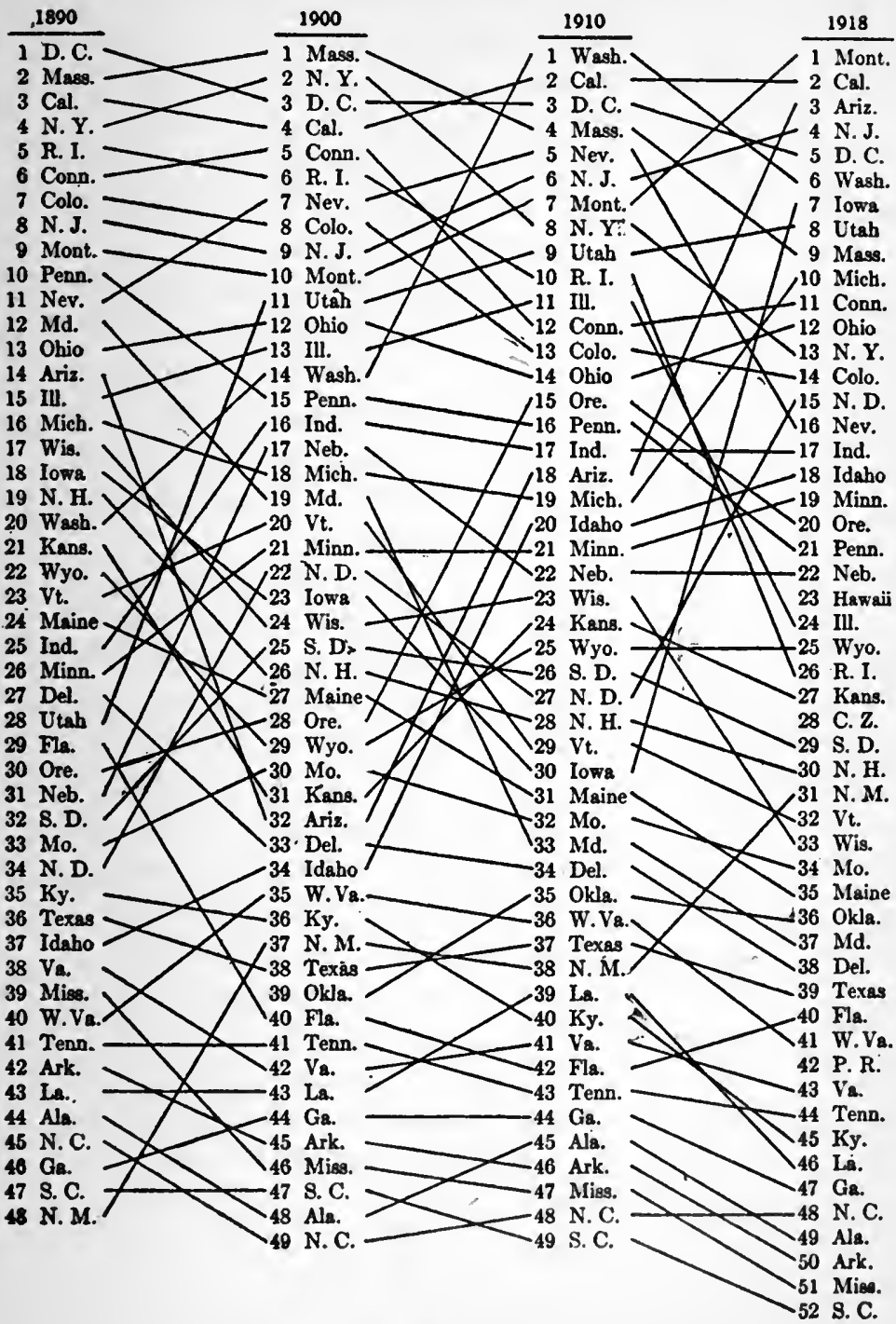


Standing of the States During Five Periods

Table 9 gives the standing of the states in ranks as indicated by their index numbers. The data are given for $1890,1900,1910,1916$, and 1918. The table presents the same data as the two preceding tables except that the figures for 1916 are also included in this table. The states are given in alphabetical order in order to facilitate ready reference.

The period from 1916 to 1918 was one of sharply rising prices and wages. The changes brought about by the resulting readjustments in state educational budgets are reflected in the sharp changes between 1916 and 1918 . In the main, the changes upward are primarily caused by sudden increases in appropriations, and the losses in rank are similarly accounted for by the failure of some states to increase school expenditures as costs and wages went up.

Still other changes appear to be related to the influence of the war on high school attendance. During the period from 1916 to 1918 the attendance in high schools increased notably, but the ratio of boys to girls fell off greatly, apparently reflecting the increased demand for labor and the consequent opportunities for earning high wages. 
TABLE 9.-RANKS OF STATES AS SHOWN BY INDEX NUMBERS FOR FIVE PERIODS

\begin{tabular}{|c|c|c|c|c|c|}
\hline & 1890 & 1900 & 1910 & 1916 & 1918 \\
\hline $\begin{array}{l}\text { Alabama } \\
\text { Arizons } \\
\text { Arkansas } \\
\text { California } \\
\text { Canal Zone } \\
\text { Colorado } \\
\text { Connecticut } \\
\text { Distriot of Columbia } \\
\text { Delaware } \\
\text { Florida }\end{array}$ & $\begin{array}{c}44 \\
14 \\
42 \\
3 \\
7 \\
6 \\
1 \\
27 \\
29\end{array}$ & $\begin{array}{c}48 \\
32 \\
45 \\
4 \\
\dddot{8} \\
5 \\
3 \\
33 \\
40\end{array}$ & $\begin{array}{c}45 \\
18 \\
46 \\
2 \\
13 \\
12 \\
3 \\
34 \\
42\end{array}$ & $\begin{array}{c}46 \\
4 \\
43 \\
1 \\
19 \\
8 \\
2 \\
35 \\
50\end{array}$ & $\begin{array}{c}49 \\
3 \\
50 \\
2 \\
28 \\
14 \\
11 \\
5 \\
38 \\
40\end{array}$ \\
\hline $\begin{array}{l}\text { Georgia } \\
\text { Hawaii } \\
\text { Idaho } \\
\text { Illinois } \\
\text { Indiana } \\
\text { Iowa } \\
\text { Kansas } \\
\text { Kentucky } \\
\text { Louisiana } \\
\text { Maine }\end{array}$ & $\begin{array}{l}46 \\
3 \dot{37} \\
15 \\
25 \\
18 \\
21 \\
35 \\
43 \\
24\end{array}$ & $\begin{array}{l}44 \\
34 \\
13 \\
16 \\
23 \\
31 \\
36 \\
43 \\
27\end{array}$ & $\begin{array}{l}44 \\
2 \dot{20} \\
11 \\
17 \\
30 \\
24 \\
40 \\
39 \\
31\end{array}$ & $\begin{array}{l}45 \\
1 \dot{14} \\
21 \\
16 \\
23 \\
24 \\
39 \\
42 \\
30\end{array}$ & $\begin{array}{l}47 \\
23 \\
18 \\
24 \\
17 \\
7 \\
27 \\
45 \\
46 \\
35\end{array}$ \\
\hline $\begin{array}{l}\text { Maryland } \\
\text { Massachusetts } \\
\text { Michigan } \\
\text { Minnesota } \\
\text { Mississippi } \\
\text { Missouri } \\
\text { Montana } \\
\text { Nebraska } \\
\text { Nevada } \\
\text { New Hampshire }\end{array}$ & $\begin{array}{c}12 \\
2 \\
16 \\
26 \\
39 \\
33 \\
9 \\
31 \\
11 \\
19\end{array}$ & $\begin{array}{l}19 \\
1 \\
18 \\
21 \\
46 \\
30 \\
10 \\
17 \\
7 \\
26\end{array}$ & $\begin{array}{c}33 \\
4 \\
19 \\
21 \\
47 \\
32 \\
7 \\
22 \\
5 \\
28\end{array}$ & $\begin{array}{l}34 \\
7 \\
18 \\
20 \\
49 \\
32 \\
3 \\
25 \\
10 \\
31\end{array}$ & $\begin{array}{l}37 \\
9 \\
10 \\
19 \\
51 \\
34 \\
1 \\
22 \\
16 \\
30\end{array}$ \\
\hline $\begin{array}{l}\text { New Jersey } \\
\text { New Mexico } \\
\text { New York } \\
\text { North Carolina } \\
\text { North Dakota } \\
\text { Ohio } \\
\text { Oklahoma } \\
\text { Oregon } \\
\text { Pennsylvanis } \\
\text { Porto Rico }\end{array}$ & $\begin{array}{c}8 \\
48 \\
4 \\
45 \\
34 \\
13 \\
30 \\
10 \\
\cdots\end{array}$ & $\begin{array}{c}9 \\
37 \\
2 \\
49 \\
22 \\
12 \\
39 \\
28 \\
15 \\
\cdots\end{array}$ & $\begin{array}{c}6 \\
38 \\
8 \\
48 \\
27 \\
14 \\
35 \\
15 \\
16 \\
\cdots\end{array}$ & $\begin{array}{l}5 \\
33 \\
12 \\
47 \\
22 \\
11 \\
37 \\
17 \\
15 \\
44\end{array}$ & $\begin{array}{l}4 \\
31 \\
13 \\
48 \\
15 \\
12 \\
36 \\
20 \\
21 \\
42\end{array}$ \\
\hline $\begin{array}{l}\text { Rhode Island } \\
\text { South Carolina } \\
\text { South Dakota } \\
\text { Tennessee } \\
\text { Texas } \\
\text { Utah } \\
\text { Vermont } \\
\text { Virginia } \\
\text { Washington } \\
\text { West Virginis } \\
\text { Wisconsin } \\
\text { Wyoming }\end{array}$ & $\begin{array}{l}5 \\
47 \\
32 \\
41 \\
36 \\
28 \\
23 \\
38 \\
20 \\
40 \\
17 \\
22\end{array}$ & $\begin{array}{l}6 \\
47 \\
25 \\
41 \\
38 \\
11 \\
20 \\
42 \\
14 \\
35 \\
24 \\
29\end{array}$ & $\begin{array}{c}10 \\
49 \\
26 \\
43 \\
37 \\
9 \\
29 \\
41 \\
1 \\
36 \\
23 \\
25\end{array}$ & $\begin{array}{c}13 \\
48 \\
29 \\
41 \\
36 \\
9 \\
28 \\
40 \\
6 \\
38 \\
27 \\
26\end{array}$ & $\begin{array}{c}26 \\
52 \\
29 \\
44 \\
39 \\
8 \\
32 \\
43 \\
6 \\
41 \\
33 \\
25\end{array}$ \\
\hline
\end{tabular}




\section{The 48 States in 1918}

Table 10 gives the rank of each state in each element of the index in 1918. In this table only the 48 states are entered. The District of Columbia, the Canal Zone, and the insular possessions are purposely omitted.

The table makes possible something of a quick diagnosis of the situation in any state. If the rank as shown in the final column is low, the entries in the other columns will show the items responsible for the poor showing. If these items are among the last five, that fact tends to indicate that low appropriations are accountable. If they are among the first five the more serious problems would appear to be directly educational rather than financial in nature.

In the items that relate to expenditures some cases will be found in which a state ranks relatively high in the 10th element which shows the monthly salaries of the teachers, but takes a low rank in the ninth column in which the figures are based on payments for non-salary purposes. In still other cases the opposite is true. In any case the data of the table aid in ascertaining the degree to which the different rankings show a relatively even balance as between themselves. 
TABLE 10.-RANK OF EACH STATE IN EACH COMPONENT OF THE INDEX IN 1918

\begin{tabular}{|c|c|c|c|c|c|c|c|c|c|c|c|}
\hline State & 1 & 2 & 3 & 4 & 5 & 6 & 7 & 8 & 9 & 10 & Index \\
\hline $\begin{array}{l}\text { Ala. } \\
\text { Ariz. } \\
\text { Ark. } \\
\text { Cal. } \\
\text { Colo. } \\
\text { Conn. } \\
\text { Del. } \\
\text { Fla. } \\
\text { Ga. } \\
\text { Idaho }\end{array}$ & \begin{tabular}{|c|c|}
43 \\
12 \\
31 \\
4 \\
21 \\
10 \\
41 \\
35 \\
37 \\
14
\end{tabular} & $\begin{array}{c}46 \\
15 \\
45 \\
3 \\
22 \\
5 \\
35 \\
43 \\
40 \\
28\end{array}$ & $\begin{array}{l}45 \\
28 \\
47 \\
13 \\
22 \\
6 \\
26 \\
44 \\
40 \\
34\end{array}$ & $\begin{array}{l}42 \\
33 \\
46 \\
1 \\
13 \\
22 \\
28 \\
40 \\
43 \\
16\end{array}$ & $\begin{array}{c}28 \\
5 \\
34 \\
14 \\
22 \\
9 \\
21 \\
41 \\
19 \\
23\end{array}$ & $\begin{array}{c}44 \\
2 \\
45 \\
4 \\
10 \\
26 \\
35 \\
36 \\
46 \\
11\end{array}$ & $\begin{array}{c}47 \\
3 \\
44 \\
2 \\
11 \\
20 \\
38 \\
34 \\
46 \\
8\end{array}$ & $\begin{array}{c}45 \\
3 \\
47 \\
2 \\
19 \\
10 \\
35 \\
37 \\
44 \\
16\end{array}$ & $\begin{array}{c}44 \\
6 \\
46 \\
16 \\
13 \\
25 \\
37 \\
33 \\
47 \\
9\end{array}$ & $\begin{array}{c}44 \\
2 \\
46 \\
1 \\
11 \\
8 \\
28 \\
40 \\
38 \\
16\end{array}$ & $\begin{array}{c}45 \\
3 \\
46 \\
2 \\
13 \\
10 \\
35 \\
37 \\
43 \\
17\end{array}$ \\
\hline $\begin{array}{l}\text { Ill. } \\
\text { Ind. } \\
\text { Iowa } \\
\text { Kans. } \\
\text { Ky. } \\
\text { La. } \\
\text { Maine } \\
\text { Mass. } \\
\text { Md. } \\
\text { Mich. }\end{array}$ & $\begin{array}{c}13 \\
3 \\
7 \\
20 \\
45 \\
48 \\
11 \\
16 \\
44 \\
6\end{array}$ & $\begin{array}{c}20 \\
8 \\
4 \\
18 \\
42 \\
47 \\
11 \\
13 \\
34 \\
7\end{array}$ & $\begin{array}{c}29 \\
31 \\
7 \\
15 \\
36 \\
42 \\
21 \\
9 \\
18 \\
15\end{array}$ & $\begin{array}{c}26 \\
17 \\
9 \\
3 \\
39 \\
38 \\
6 \\
7 \\
34 \\
20\end{array}$ & $\begin{array}{c}12 \\
4 \\
32 \\
36 \\
39 \\
46 \\
20 \\
8 \\
45 \\
13\end{array}$ & $\begin{array}{l}27 \\
28 \\
8 \\
22 \\
39 \\
40 \\
33 \\
19 \\
34 \\
18\end{array}$ & $\begin{array}{l}24 \\
17 \\
5 \\
22 \\
40 \\
42 \\
32 \\
19 \\
37 \\
10\end{array}$ & $\begin{array}{c}9 \\
11 \\
25 \\
28 \\
43 \\
36 \\
39 \\
8 \\
27 \\
15\end{array}$ & $\begin{array}{c}26 \\
18 \\
3 \\
19 \\
40 \\
42 \\
34 \\
24 \\
36 \\
10\end{array}$ & $\begin{array}{c}7 \\
22 \\
33 \\
31 \\
41 \\
34 \\
43 \\
6 \\
12 \\
21\end{array}$ & $\begin{array}{c}22 \\
16 \\
6 \\
25 \\
41 \\
42 \\
32 \\
8 \\
34 \\
9\end{array}$ \\
\hline $\begin{array}{l}\text { Minn. } \\
\text { Miss. } \\
\text { Mo. } \\
\text { Mont. } \\
\text { Neb. } \\
\text { Nev. } \\
\text { N. C. } \\
\text { N. D. } \\
\text { N. H. } \\
\text { N. J. }\end{array}$ & $\begin{array}{c}17 \\
33 \\
23 \\
1 \\
15 \\
25 \\
34 \\
28 \\
32 \\
22\end{array}$ & $\begin{array}{l}19 \\
36 \\
26 \\
1 \\
14 \\
23 \\
44 \\
30 \\
27 \\
12\end{array}$ & $\begin{array}{c}20 \\
41 \\
25 \\
33 \\
24 \\
16 \\
46 \\
23 \\
12 \\
4\end{array}$ & $\begin{array}{c}15 \\
47 \\
25 \\
23 \\
12 \\
8 \\
44 \\
31 \\
5 \\
21\end{array}$ & $\begin{array}{l}37 \\
38 \\
31 \\
40 \\
33 \\
10 \\
25 \\
47 \\
15 \\
2\end{array}$ & $\begin{array}{c}14 \\
48 \\
31 \\
1 \\
13 \\
7 \\
47 \\
3 \\
20 \\
9\end{array}$ & $\begin{array}{c}16 \\
48 \\
30 \\
1 \\
13 \\
7 \\
45 \\
6 \\
26 \\
12\end{array}$ & $\begin{array}{c}17 \\
48 \\
23 \\
6 \\
22 \\
24 \\
46 \\
20 \\
30 \\
1\end{array}$ & $\begin{array}{c}8 \\
48 \\
31 \\
1 \\
11 \\
20 \\
45 \\
2 \\
21 \\
7\end{array}$ & $\begin{array}{l}20 \\
45 \\
19 \\
15 \\
25 \\
14 \\
48 \\
24 \\
29 \\
5\end{array}$ & $\begin{array}{c}18 \\
47 \\
31 \\
1 \\
21 \\
15 \\
44 \\
14 \\
27 \\
4\end{array}$ \\
\hline $\begin{array}{l}\text { N. M. } \\
\text { N. Y. } \\
\text { Ohio } \\
\text { Okla. } \\
\text { Ore. } \\
\text { Penn. } \\
\text { R. I. } \\
\text { S. C. } \\
\text { S. D. } \\
\text { Tenn. }\end{array}$ & $\begin{array}{c}46 \\
27 \\
5 \\
42 \\
8 \\
24 \\
38 \\
40 \\
47 \\
19\end{array}$ & $\begin{array}{c}39 \\
16 \\
9 \\
37 \\
2 \\
21 \\
24 \\
48 \\
31 \\
32\end{array}$ & $\begin{array}{c}32 \\
2 \\
27 \\
30 \\
5 \\
10 \\
1 \\
48 \\
3 \\
39\end{array}$ & $\begin{array}{l}41 \\
18 \\
19 \\
32 \\
11 \\
29 \\
24 \\
48 \\
14 \\
45\end{array}$ & $\begin{array}{c}16 \\
1 \\
7 \\
35 \\
24 \\
11 \\
6 \\
48 \\
44 \\
27\end{array}$ & $\begin{array}{l}12 \\
16 \\
21 \\
32 \\
23 \\
24 \\
25 \\
42 \\
6 \\
43\end{array}$ & $\begin{array}{l}27 \\
23 \\
15 \\
33 \\
18 \\
25 \\
29 \\
43 \\
21 \\
41\end{array}$ & \begin{tabular}{|c|}
18 \\
14 \\
13 \\
31 \\
21 \\
5 \\
12 \\
42 \\
32 \\
41
\end{tabular} & $\begin{array}{c}4 \\
30 \\
17 \\
32 \\
28 \\
14 \\
23 \\
41 \\
5 \\
43\end{array}$ & \begin{tabular}{|c|}
27 \\
4 \\
18 \\
26 \\
17 \\
13 \\
10 \\
47 \\
32 \\
42
\end{tabular} & $\begin{array}{l}28 \\
12 \\
11 \\
33 \\
19 \\
20 \\
24 \\
48 \\
26 \\
40\end{array}$ \\
\hline $\begin{array}{l}\text { Texas } \\
\text { Utah } \\
\text { Va. } \\
\text { Vt. } \\
\text { Wash. } \\
\text { Wis. } \\
\text { W. Va. } \\
\text { Wyo. }\end{array}$ & $\begin{array}{c}26 \\
9 \\
39 \\
18 \\
29 \\
36 \\
30 \\
2\end{array}$ & $\begin{array}{l}33 \\
10 \\
41 \\
17 \\
25 \\
29 \\
38 \\
6\end{array}$ & $\begin{array}{c}37 \\
19 \\
38 \\
17 \\
11 \\
8 \\
43 \\
35\end{array}$ & $\begin{array}{c}36 \\
27 \\
35 \\
4 \\
2 \\
10 \\
37 \\
30\end{array}$ & $\begin{array}{c}29 \\
3 \\
43 \\
18 \\
30 \\
17 \\
26 \\
42\end{array}$ & $\begin{array}{l}38 \\
17 \\
41 \\
29 \\
5 \\
30 \\
37 \\
15\end{array}$ & $\begin{array}{c}35 \\
14 \\
39 \\
28 \\
9 \\
31 \\
36 \\
4\end{array}$ & $\begin{array}{c}33 \\
7 \\
40 \\
34 \\
4 \\
26 \\
38 \\
29\end{array}$ & $\begin{array}{l}35 \\
15 \\
39 \\
29 \\
12 \\
27 \\
38 \\
22\end{array}$ & $\begin{array}{c}35 \\
\mathbf{9} \\
\mathbf{3 9} \\
\mathbf{3 6} \\
3 \\
30 \\
\mathbf{3 7} \\
\mathbf{2 3}\end{array}$ & $\begin{array}{c}\mathbf{3 6} \\
\mathbf{7} \\
\mathbf{3 9} \\
\mathbf{2 9} \\
\mathbf{5} \\
\mathbf{3 0} \\
\mathbf{3 8} \\
\mathbf{2 3}\end{array}$ \\
\hline
\end{tabular}




\section{Gains and Losses in 28 Years}

Table 11 is similar to Table 10 in that it gives data for the 48 states only. The first four columns show, to the nearest whole number, the gains or losses in the index number in each period and for the whole 28 years from 1890 to 1918 . In the same way the figures in the second set of columns show the gains or losses in the ranks of the states in each of the three periods and during the whole 28 years. Since the numbers of the first four columns are stated as whole numbers and without their decimal fractions, the figures in the fourth column do not in every case appear to be in accord with those in the first three columns. They are, however, accurately recorded.

It is noteworthy that there have been but few cases in which the index number of a state has actually fallen. The differences between the states are mostly those of degree.

During the 28 years the smallest gain in the index number has been that of Mississippi, of eight points, and the greatest that of New Mexico, which has gone forward 43 points. The extremes for the changes of rank are found in the cases of Maryland, which has lost 25 places, and Utah, which has gained 20. The figures for Oklahoma cover only the period from 1900 to 1918 . 
TABLE 11.-CHANGES IN INDEX NUMBERS AND IN RANKS OF STATES. 1890 to 1918

\begin{tabular}{|c|c|c|c|c|c|c|c|c|}
\hline & \multicolumn{4}{|c|}{ Changes in Index } & \multicolumn{4}{|c|}{ Changes in Rank } \\
\hline & $\begin{array}{c}1890 \\
\text { to } \\
1900\end{array}$ & $\begin{array}{c}1900 \\
\text { to } \\
1910\end{array}$ & $\begin{array}{c}1910 \\
\text { to } \\
1918\end{array}$ & $\begin{array}{c}1890 \\
\text { to } \\
1918\end{array}$ & $\begin{array}{c}1890 \\
\text { to } \\
1900\end{array}$ & $\begin{array}{c}1900 \\
\text { to } \\
1910\end{array}$ & $\begin{array}{c}1910 \\
\text { to } \\
1918\end{array}$ & $\begin{array}{c}1890 \\
\text { to } \\
1918\end{array}$ \\
\hline $\begin{array}{l}\text { Ala. } \\
\text { Ariz. } \\
\text { Ark. } \\
\text { Cal. } \\
\text { Colo. } \\
\text { Conn. } \\
\text { Del. } \\
\text { Fla. } \\
\text { Ga. } \\
\text { Idaho }\end{array}$ & $\begin{array}{l}+2 \\
-3 \\
+1 \\
+4 \\
+4 \\
+1 \\
+7 \\
+6 \\
+6\end{array}$ & $\begin{array}{l}+7 \\
+16 \\
+6 \\
+16 \\
+7 \\
+6 \\
+8 \\
+8 \\
+7 \\
+16\end{array}$ & $\begin{array}{l}+4 \\
+20 \\
+3 \\
+11 \\
+10 \\
+11 \\
+4 \\
+8 \\
+4 \\
+14\end{array}$ & $\begin{array}{l}+13 \\
+33 \\
+10 \\
+27 \\
+21 \\
+21 \\
+13 \\
+9 \\
+17 \\
+36\end{array}$ & $\begin{array}{l}-4 \\
-18 \\
-3 \\
-1 \\
-1 \\
+1 \\
-6 \\
-11 \\
+2 \\
+3\end{array}$ & $\begin{array}{l}+3 \\
+14 \\
-1 \\
+2 \\
-5 \\
-7 \\
-1 \\
-2 \\
\ddot{+14}\end{array}$ & $\begin{array}{l}-4 \\
+15 \\
-4 \\
- \\
-1 \\
+1 \\
-4 \\
+2 \\
-3 \\
+2\end{array}$ & $\begin{array}{l}-5 \\
+11 \\
-8 \\
+1 \\
-7 \\
-5 \\
-11 \\
-11 \\
-1 \\
+19\end{array}$ \\
\hline $\begin{array}{l}\text { Ill. } \\
\text { Ind. } \\
\text { Iowa } \\
\text { Kans. } \\
\text { Ky. } \\
\text { La. } \\
\text { Maine } \\
\text { Mass. } \\
\text { Md. } \\
\text { Mich. }\end{array}$ & & $\begin{array}{l}+13 \\
+10 \\
+7 \\
+11 \\
+5 \\
+9 \\
+6 \\
+6 \\
+3 \\
+9\end{array}$ & $\begin{array}{l}+7 \\
+13 \\
+21 \\
+12 \\
+5 \\
+3 \\
+7 \\
+5 \\
+5 \\
+15\end{array}$ & $\begin{array}{l}+25 \\
+29 \\
+31 \\
+24 \\
+12 \\
+16 \\
+17 \\
+15 \\
+10 \\
+28\end{array}$ & $\begin{array}{l}+2 \\
+9 \\
-5 \\
-10 \\
-1 \\
\because 3 \\
+1 \\
-7 \\
-2\end{array}$ & $\begin{array}{l}+2 \\
-1 \\
-7 \\
+7 \\
-4 \\
+4 \\
-4 \\
-3 \\
-14 \\
-1\end{array}$ & $\begin{array}{l}-13 \\
\dot{+} 23 \\
-3 \\
-5 \\
-7 \\
-4 \\
-5 \\
-4 \\
+9\end{array}$ & $\begin{array}{l}-9 \\
+8 \\
+11 \\
-6 \\
-10 \\
-3 \\
-11 \\
-7 \\
-25 \\
+6\end{array}$ \\
\hline $\begin{array}{l}\text { Minn. } \\
\text { Miss. } \\
\text { Mo. } \\
\text { Mont. } \\
\text { Neb. } \\
\text { Nev. } \\
\text { N. C. } \\
\text { N. D. } \\
\text { N. H. } \\
\text { N. J. }\end{array}$ & $\begin{array}{l}+6 \\
-1 \\
+6 \\
+4 \\
+10 \\
+8 \\
\ddot{+10} \\
+3 \\
+3\end{array}$ & $\begin{array}{l}+10 \\
+5 \\
+7 \\
+14 \\
+8 \\
+14 \\
+8 \\
+7 \\
+8 \\
+14\end{array}$ & $\begin{array}{l}+13 \\
+4 \\
+11 \\
+22 \\
+13 \\
+3 \\
+5 \\
+17 \\
+12 \\
+12\end{array}$ & $\begin{array}{l}+29 \\
+8 \\
+24 \\
+40 \\
+31 \\
+25 \\
+13 \\
+34 \\
+23 \\
+29\end{array}$ & $\begin{array}{l}+5 \\
-7 \\
+3 \\
-1 \\
+14 \\
+4 \\
-4 \\
+12 \\
-7 \\
-1\end{array}$ & $\begin{array}{l}-1 \\
-2 \\
+3 \\
-5 \\
+2 \\
+1 \\
-5 \\
-2 \\
+3\end{array}$ & $\begin{array}{l}+2 \\
-4 \\
-2 \\
+6 \\
\because 11 \\
\ddot{+12} \\
-2 \\
+2\end{array}$ & $\begin{array}{l}+7 \\
-12 \\
-1 \\
+8 \\
+9 \\
-5 \\
-3 \\
+19 \\
-11 \\
+4\end{array}$ \\
\hline $\begin{array}{l}\text { N. M. } \\
\text { N. Y. } \\
\text { Ohio } \\
\text { Okla. } \\
\text { Ore. } \\
\text { Penn. } \\
\text { R. I. } \\
\text { S. C.. } \\
\text { S. D. } \\
\text { Tenn. }\end{array}$ & $\begin{array}{l}+4 \\
+2 \\
+4 \\
+9 \\
+8 \\
+1\end{array}$ & $\begin{array}{l}+6 \\
+5 \\
+12 \\
+13 \\
+16 \\
+10 \\
+8 \\
+4 \\
+9 \\
+7\end{array}$ & $\begin{array}{l}+22 \\
+7 \\
+11 \\
+8 \\
+10 \\
+11 \\
+5 \\
+4 \\
+12 \\
+6\end{array}$ & $\begin{array}{r}+43 \\
+18 \\
+27 \\
+21 \\
+30 \\
+23 \\
+17 \\
+17 \\
+29 \\
+14\end{array}$ & $\begin{array}{l}+11 \\
+2 \\
+1 \\
+2 \\
-5 \\
-1 \\
+7\end{array}$ & $\begin{array}{l}-1 \\
-6 \\
-2 \\
+4 \\
+13 \\
-1 \\
-4 \\
-2 \\
-1 \\
-2\end{array}$ & $\begin{array}{l}+7 \\
-5 \\
+2 \\
-1 \\
-5 \\
-5 \\
-16 \\
-3 \\
-3 \\
-1\end{array}$ & $\begin{array}{l}+17 \\
-9 \\
+1 \\
+3 \\
+10 \\
-11 \\
-21 \\
-5 \\
+3 \\
-3\end{array}$ \\
\hline $\begin{array}{l}\text { Texas } \\
\text { Utah } \\
\text { V8. } \\
\text { Vt. } \\
\text { Wash. } \\
\text { Wis. } \\
\text { W. Va. } \\
\text { Wyo. }\end{array}$ & $\begin{array}{l}+5 \\
+6 \\
+3 \\
+5 \\
+2\end{array}$ & $\begin{array}{l}+8 \\
+13 \\
+8 \\
+7 \\
+24 \\
+9 \\
+6 \\
+11\end{array}$ & $\begin{array}{l}+9 \\
+10 \\
+5 \\
+10 \\
+3 \\
+8 \\
+5 \\
+14\end{array}$ & $\begin{array}{l}+18 \\
+32 \\
+13 \\
+22 \\
+33 \\
+20 \\
+16 \\
+27\end{array}$ & $\begin{array}{l}-2 \\
+17 \\
-4 \\
+3 \\
+6 \\
-7 \\
+5 \\
-7\end{array}$ & $\begin{array}{l}+1 \\
+2 \\
+1 \\
-9 \\
+13 \\
+1 \\
-1 \\
+4\end{array}$ & $\begin{array}{l}-2 \\
+1 \\
-2 \\
-3 \\
-5 \\
-10 \\
-5 \\
\cdots\end{array}$ & $\begin{array}{l}-3 \\
+20 \\
-5 \\
-9 \\
+14 \\
-16 \\
-1 \\
-3\end{array}$ \\
\hline
\end{tabular}




\section{Index Numbers Stated as Whole}

NuMBERS

In Table 12 the index numbers for 1918 for the 48 states, the District of Columbia, and the territorial possessions are given as whole numbers. That is, the decimal fractions are omitted and the figures given to the nearest whole number. The data of this table give in some ways a more accurate picture of the conditions shown by the index numbers than do the previous tables in which the fractions are retained.

They show that considerable groups of states are virtually tied in the final results. Thus there are six states with a score of 59, four with one of 57 , three with the number 55 , and so on.

They show further that in a general way the states tend to fall into two great groups. One of these consists of the Southern states with index numbers averaging around 35, while the other group consists of all the remaining states with indexes averaging around 58. If the numbers for all the states are plotted on a surface of distribution this tendency of the index numbers to form two groups is clearly manifested. 
TABLE 12.-INDEX NUMBERS OF STATES IN 1918

\begin{tabular}{|c|c|c|c|}
\hline $\begin{array}{l}\text { Montana } \\
\text { California } \\
\text { Arizona } \\
\text { New Jersey } \\
\text { District of Columbia } \\
\text { Washington } \\
\text { Iowa } \\
\text { Utah } \\
\text { Massachusetts } \\
\text { Michigan } \\
\text { Connecticut } \\
\text { Ohio } \\
\text { New York } \\
\text { Colorado } \\
\text { North Dakota } \\
\text { Nevada } \\
\text { Indiana } \\
\text { Idaho } \\
\text { Minnesota } \\
\text { Oregon } \\
\text { Pennsylvania } \\
\text { Nebrasks } \\
\text { Hawaii } \\
\text { Illinois } \\
\text { Wyoming } \\
\text { Rhode Island }\end{array}$ & . & $\begin{array}{l}76 \\
71 \\
66 \\
66 \\
64 \\
64 \\
62 \\
61 \\
61 \\
60 \\
60 \\
60 \\
59 \\
59 \\
59 \\
59 \\
59 \\
59 \\
58 \\
58 \\
58 \\
57 \\
57 \\
57 \\
57 \\
56\end{array}$ & $\begin{array}{l}\text { Kansas } \\
\text { Canal Zone } \\
\text { South Dakota } \\
\text { New Hampshire } \\
\text { New Mexico } \\
\text { Vermont } \\
\text { Wisconsin } \\
\text { Missouri } \\
\text { Maine } \\
\text { Oklahoma } \\
\text { Maryland } \\
\text { Delaware } \\
\text { Texas } \\
\text { Florida } \\
\text { West Virginia } \\
\text { Porto Rico } \\
\text { Virginia } \\
\text { Tennessee } \\
\text { Kentucky } \\
\text { Louisiana } \\
\text { Georgia } \\
\text { North Carolina } \\
\text { Alabama } \\
\text { Arkansas } \\
\text { Mississippi } \\
\text { South Carolina }\end{array}$ \\
\hline
\end{tabular}

\section{Educational Results and Financial} EXPENDITURES

Table 13 gives two sets of figures for each state for 1918. The first of these is the sum of the five components of the index number that are educational and not financial in nature. These items relate to the school term and the children but not to the expenditures. The second figure for each state is the sum of the five financial components. All the figures are stated as whole numbers with the decimal places omitted. The states are placed in the descending order of the combined educational items, which are those in the first column. 
There are two significant features shown by this table. The first is that there is a generally close agreement between the educational figures in the first column and the financial ones in the second column in the matter of relative magnitude. In general the high educational figures are accompanied by high financial ones, and the low educational totals by correspondingly low financial ones. This is one way of saying that a state having a long school term, high attendance, and many children in high school must pay more for the upkeep of its educational system than one which has none of these advantages.

Another interpretation of the figures, which also is valid, is that the figures for school expenditures do have a close relation to those which show the amount of education given and tell how many are in high school, and that they are important indicators of the efficiency of the system and the quality of the education the children receive.

The other important fact about the figures is that in general the educational figures in the first column are larger than the financial figures in the second column. The average relationship is approximately that of four to three. However, since there is a greater range 
of variation in the financial items, and since both sets are inter-related and in some measure inter-dependent, neither set dominates the other in final effect.

The coefficient of correlation between the two sets of figures is high. As computed by Pearson's formula it is .78.

TABLE 13.-SUM OF FIVE EDUCATIONAL COMPONENTS AND SUM OF FIVE FINANCIAL COMPONENTS OF INDEX NUMBER FOR EACH STATE IN 1918

\begin{tabular}{|c|c|c|c|c|c|}
\hline State & $\begin{array}{c}\text { Sum of } \\
\text { educations] } \\
\text { components }\end{array}$ & $\begin{array}{c}\text { Sum of } \\
\text { financial } \\
\text { components }\end{array}$ & State & $\begin{array}{c}\text { Sum of } \\
\text { educational } \\
\text { components }\end{array}$ & $\begin{array}{c}\text { Sum of } \\
\text { financial } \\
\text { components }\end{array}$ \\
\hline $\begin{array}{l}\text { Cal. } \\
\text { Ore. } \\
\text { Iowa } \\
\text { Ind. } \\
\text { Conn. } \\
\text { Mont. } \\
\text { Mass. } \\
\text { Mich. } \\
\text { N. Y. } \\
\text { N. J. } \\
\text { Ohio } \\
\text { Maine }\end{array}$ & $\begin{array}{l}355 \\
\mathbf{3 3 2} \\
\mathbf{3 3 0} \\
\mathbf{3 2 9} \\
\mathbf{3 2 8} \\
\mathbf{3 2 8} \\
327 \\
327 \\
325 \\
325 \\
323 \\
322\end{array}$ & $\begin{array}{l}358 \\
246 \\
289 \\
259 \\
269 \\
430 \\
283 \\
277 \\
269 \\
335 \\
274 \\
151\end{array}$ & $\begin{array}{l}\text { Ill. } \\
\text { Wis. } \\
\text { Minn. } \\
\text { Idaho } \\
\text { Mo. } \\
\text { S. D. } \\
\text { N. D. } \\
\text { Del. } \\
\text { Texas } \\
\text { Okla. } \\
\text { Md. } \\
\text { Tenn. }\end{array}$ & $\begin{array}{l}302 \\
302 \\
300 \\
292 \\
288 \\
281 \\
271 \\
269 \\
261 \\
257 \\
253 \\
251\end{array}$ & $\begin{array}{l}266 \\
212 \\
284 \\
293 \\
208 \\
269 \\
321 \\
156 \\
151 \\
188 \\
179 \\
100\end{array}$ \\
\hline $\begin{array}{l}\text { Utah } \\
\text { Vt. } \\
\text { Kans. } \\
\text { Nev. } \\
\text { N. H. } \\
\text { Wash. } \\
\text { R. I. } \\
\text { Wyo. } \\
\text { Colo. } \\
\text { Neb. } \\
\text { Ariz. } \\
\text { Penn. }\end{array}$ & $\begin{array}{l}321 \\
316 \\
314 \\
312 \\
311 \\
310 \\
309 \\
305 \\
304 \\
303 \\
302 \\
302\end{array}$ & $\begin{array}{l}293 \\
199 \\
237 \\
279 \\
233 \\
327 \\
255 \\
262 \\
289 \\
268 \\
360 \\
274\end{array}$ & $\begin{array}{l}\text { N. M. } \\
\text { W. Va. } \\
\text { Ga. } \\
\text { Va. } \\
\text { Ky. } \\
\text { Miss. } \\
\text { Fla. } \\
\text { N.C. } \\
\text { Ark. } \\
\text { Ala. } \\
\text { La. } \\
\text { S. C. }\end{array}$ & $\begin{array}{l}251 \\
248 \\
241 \\
240 \\
239 \\
237 \\
231 \\
230 \\
226 \\
221 \\
213 \\
198\end{array}$ & $\begin{array}{r}280 \\
129 \\
85 \\
113 \\
110 \\
63 \\
147 \\
76 \\
77 \\
85 \\
125 \\
96\end{array}$ \\
\hline
\end{tabular}




\section{Correlations}

The coefficients of correlation have been computed between the 10 different series of components and the final series of index numbers for 1918. The purpose of these computations has been to find how nearly each series of items corresponds with the final index numbers. This process throws light on the relative value of the different elements. It does not do this in a complete or final way because the different elements are inter-related and, as each exercises some influence in determining the final index number, it is almost inevitable that some degree of correlation should exist between each one of the series and the final results. It has nevertheless been thought worth while to make the computations for purposes of comparison.

The coefficients of correlation have been computed from the ranks of Table 9 instead of from the original data, and the work has been done by the method of squared differences.

The resulting coefficients show the degree of correlation between each of the 10 series of elements and the final series of index numbers for 1918. They are as follows: 
1. Per cent of school population attending

2. Days attended per child of school age

3. Days schools were kept open

4. High school attendance

5. Per cent of boys to girls in high school

6. Expenditure per child attending

7. Expenditure per child of school age

8. Expenditure per teacher

9. Expenditure for non-salary purposes

10. Teachers' salaries

Five non-financial items combined Five financial items combined

The coefficients in general are high, as would be expected from the nature of the data. It is, however, interesting to note that there is a high relationship between the proportion of children enrolled in high schools and the final educational index. It is also interesting to note that expenditures for purposes other than teaching appear to be as highly correlated with the final index numbers as do the expenditures paid out directly for teachers' salaries.

\section{Significance of The Index Number}

Various questions as to the reliability and degree of significance of the index number call for consideration. Perhaps the most important of these relates to the degree to which the different items, separately or in combination, constitute trustworthy indicators of educational efficiency. 
If some great national agency were to undertake a survey of each of the 48 state school systems, it would surely report on many phases of their work not included in the items of the index. Among such phases would be the legal basis of the system, its organization, professional leadership and supervision, business management, course of study, teaching staff, plant and equipment, and the results of standard tests of classroom work.

These elements, and others like them, are not directly included in the index, although some of them are crudely measured by the several items for financial expenditure. The sad fact is that, if there were available such information as surveys gather on most of the subjects suggested above, it would still be impossible to state the results in terms that could be included in an index number. The reason for this is that there is as yet little exact knowledge about education. We do not know just what kind of a teaching staff does the best work, or what kind of supervision is the most effective, or how many supervisors there ought to be, or how to measure organization in quantitative terms, or how to evaluate most of the other matters on which we report in survey making. 
Under present circumstances the best that can be done in an undertaking of the present sort is to take all the items for which continuous, reliable data can be secured and to combine them by as careful and impartial methods as can be brought to bear. In the present case it is to be regretted that only 10 items could be found that seemed to meet the requirements that were laid down at the outset of the work. If the number of accurate and significant series used were greater, the reliability of the results would be enhanced.

\section{Accuracy of Data}

Fortunately the data used are of a high degree of accuracy as compared with most other sorts of mass statistics. Data for school attendance are gathered at their source daily in set forms by people who are paid for their work and they are compiled in permanent offices by paid employees. Something of the same conditions maintains with respect to figures for public school expenditures. There are few other kinds of data of a comparable sort for which so much may be claimed on the side of reliability. As compared with censuses of population, occupations, or manufactures, or government data on agriculture, or strength 
returns of the Army, Navy, and Marine Corps, the data for school attendance and gross expenditures are certainly in the lead in the matter of accuracy. As compared with data for the results of psychological tests or measurements of class room products they are almost indefinitely more reliable.

There are certain other data published in the reports of the Bureau of Education which have been excluded from the index because so much could not be claimed for them on the score of accuracy. Among these data are the figures for the enrollment of pupils. These have been carefully tested and rejected. The figures for attendance have been used instead. Other figures that have been considered, but rejected, include those for the valuation of school property and the detailed figures for expenditures for such matters as general control, textbooks, and the like.

\section{Private and Parochial Schools}

Another question that may well be raised is that relating to the procedure which takes into account only the figures for the public schools and does not allow for the enrollment in private and parochial schools. In some states the pupils in schools under private 
and church control are relatively numerous. According to the report for 1918 there were then 12 states in which the private school enrollment was more than 10 per cent as great as that of the public schools. Seven of these 12 states were in the North Atlantic group.

From a statistical point of view a state having a large proportion of children in private schools tends to be penalized in the two elements of the index which relate to the attendance in public schools, and rewarded in one of the high school elements and the five items that relate to financial expenditures. Being relieved of the expense of educating a large number of the children, such a state ought to make a better showing in the matter of expenditures for the pupils who are in public schools than it would if it had to bear the whole burden of expense. Since the differences involved tend in some measure to counterbalance each other it is not believed that they have an important bearing on the final results and no further allowance has been made for them. In so far as they do influence the results they favor the state with many children in private schools. 


\section{Different Types of States}

Another set of questions centers around the propriety of using the same index number to measure conditions in wealthy states and those with small resources, northern states and southern states, those that have large urban populations and states predominantly rural, those with many foreign-born in the population and those that have few.

The answer to these questions is that the index number attempts to measure accomplishment rather than effort. It does,not seek to award credit or apportion blame. Like the Census figures for population increase or death rate, or those of the Bureau of Labor for cost of living in different parts of the country, it attempts to measure a status rather than to explain why that status came into being.

Careful checking has been done to ascertain whether or not the results were seriously affected by the fact that some states have many more small rural schools than others and hence fewer children per teacher. The results show that these differences have no constant relationship to the index numbers. For the entire United States the number of children attending per teacher is 23 . The highest average is 
among states having the lowest index numbers. The next highest is among those having the highest index numbers and the intermediate averages among states having medium index ranks.

\section{Purchasing Power of Money}

One criticism that might be made of the use of the index number over a long period of time is that several of the elements of the index are financial in nature and that the purchasing power of money has undergone changes that have recently been increasingly marked. Careful attempts have been made to devise a satisfactory correction for this but they resulted in slight changes in the final results and serious complications in the computations. For this reason they were given up in favor of the more simple and direct method. This factor has practically no effect on the relative standing of the states at each given date.

\section{High School Data}

There are several matters with regard to the high school data that need comment. The first of these relates to the basis on which the data that have been published by the Bureau 
of Education have been gathered. They are not in reality attendance figures. They are membership figures given at the close of the school year and appear to run somewhat above the figures for average attendance and to be in general lower than the figures for total enrollment. This discrepancy affects only one item of the index and that in only slight degree.

A further comment with regard to the high school data is that the index assumes that under ideal conditions one-third of the children in school might be found in the high schools. The basis for this assumption is that there are commonly four high school classes and eight elementary grades so that the high school course is one-third of the entire course. While this is true, it does not follow that one-third of the pupils could normally be found in the high school even under ideal conditions. The reasons for this are that many children die before reaching the high school age and, in our increasing populations, there are always further additional numbers of children in the lower age groups. On the other hand, many children enter high school from private and parochial schools which tends to offset the differences 
just mentioned. In the data for recent years care has been taken to distribute the pupils in junior high schools in the grades they would have been in on the conventional basis of organization.

\section{'Teachers' Salaries}

The monthly salaries of teachers have been computed by dividing the total expenditures for salaries of teachers by the total number of teachers employed, and dividing this result by 12 . The methods of gathering these data have not remained constant during the time covered by the reports of the Bureau of Education. In recent years the figures include principals and supervisory officers as well as teachers but, since the salaries of these officers are also included, the per capita figures are but slightly affected by the changes in the methods of gathering the original data. Another important matter with respect to these data is that the figures used in the present computations are based on the whole number of different teachers employed, rather than on the number of teaching positions. This method tends to penalize the states that have the greatest turnovers in the teaching force. If a state employs many more teachers 
during one year than it has teaching positions, the resulting payments per teacher will be reduced. In the computations for the index number the figures giving the total number of different teachers employed during the year have been used because there is good evidence that they are more uniformly and more accurately reported by the different states than are the data for teaching positions. The difference in method makes the figures used in the index differ somewhat from the corresponding salary figures published by the Bureau of Education.

\section{Limitations}

The index number that has been described should not be used in the attempt to measure or compare conditions in the school systems of cities. It is valid for large geographical units to a degree that it would not be for small ones. The principal reason for this difference is to be found in the greater stability of the data for the large unit composed of a great number of small units acting almost independently of each other.

Perhaps the best illustration of this is found in the figures for expenditures. Cities commonly defray the expenses of erecting new 
buildings by issuing bonds and these issues are often used to pay for the structures called for by an extensive building program. The result is that educational expenditures in cities are subject to large fluctuations. Sometimes they are nearly doubled for a period of two or three years and then fall back to close to their former level.

Under these conditions the use of the present index number would give misleading results if computed for cities. The figures for an entire state are very different for the reason that the state is made up of many cities and towns which make their exceptional expenditures at different times so that the sum total moves forward from year to year with comparative steadiness.

Observations of a somewhat similar sort may be made with respect to the figures giving the number of children of school age and those showing the attendance in public schools. The data for school population are more reliable for the states than they are for cities in inter-census years. Those for attendance in public schools are less affected by attendance in private and parochial schools, when taken for an entire state, than they would be if 
they were for the component sub-divisions of the state.

For all of the foregoing reasons the index number that has been presented should not be used to measure city school systems. An index number for city systems would have to be constructed from different elements and after most careful tests to identify the items that could be used as trustworthy indicators of school efficiency in the municipalities.

\section{Summary}

This volume presents an index number for measuring the effectiveness of state school systems. It is a composite of 10 different elements, of which five are measures of the amount of education received by the children, and the remaining five are measures of the expenditures made to purchase this education.

The index number has been computed by methods similar in principle to those in current use for measuring the fluctuations in the prices of commodities, the cost of living, the value of securities, and the like. It has been computed for the entire United States for each year from 1871 to 1918, and for each state for the years 1890, 1900, 1910, 1916 and 
1918. It has also been computed for the District of Columbia for each of these years and for Hawaii, the Canal Zone, and Porto Rico for 1918 .

In constructing the index number the purpose has been to use as component elements all the series of measures that were available in substantially uniform and reliable records for the entire period of 47 years, that were in general as fair to each state as to each other state, and that were of such a nature that increases in them reflected improved educational conditions, and decreases in them reflected poorer educational conditions.

It is the belief of the author that this index number is relatively reliable as an indicator of changes in the effectiveness of public education from year to year in the country as a whole over the period included in the present report. In the cases of the individual states it gives significantly valuable information as to changes in absolute and relative status over periods of several years, such as decades. The minute differences in the decimal places of the numbers for the different states should not be regarded as significant.

In general the index number should be regarded as reliable rather than precise. Its 
methods are rigid and impartial, but not considerate or interpretative. It measures results without considering causes. The purpose of the index number is to make it possible for state school systems to measure their progress from year to year and to compare their attainments with those of their neighbors. 


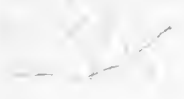

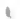



This book is DUE on the last date stamped below

RESERTA는 1954 RESERVE JUN 1954

\section{RESERVE SEP 1954}

APR 301955

RESERVE FALL $195 \%$

FEB 2 3 1957

MAY 11959

R. SEIVED

DEC 201969

DEC ? ? 1959

은

$$
\text { RECEIVED }
$$

JAN 121981

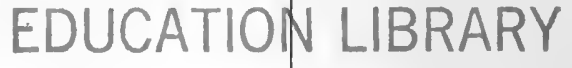

JAN 12 '81 -9 PM

L4II - U.1 LIB.

MAR 301979

RECEIVED

APR 11971

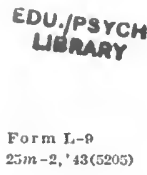

UNI VERSITY OF CALIFUKNIA

\section{$A T$}

LOS ANGELES

LIBRARY 


\section{IA \\ 219 \\ Education \\ Library}


